\title{
Abel transform and integrals of Bessel local times
}

\author{
by \\ Mihai Gradinaru ${ }^{1}$, Bernard Roynette, Pierre Vallois \\ Institut de Mathématiques Elie Cartan, Université Henri Poincaré, \\ B.P. 239, 54506 Vandouvre-lès-Nancy Cedex, France \\ and \\ Marc Yor \\ Laboratoire de Probabilités, Université Pierre et Marie Curie, \\ Tour 56 (3ème étage), 4, place Jussieu, 75252 Paris Cedex, France
}

ABstract. - We study integrals of the type $\int_{0}^{t} \varphi(s) d \mathrm{~L}_{s}$, where $\varphi$ is a positive locally bounded Borel function and $\mathrm{L}_{t}$ denotes the local time at level 0 of a Bessel process of dimension $d, 0<d<2$.

1991 Mathematics Subject Classifications : 60J65, 60J55, 45E10.

Keywords : Bessel local time, Abel's integral operator.

RÉSUmÉ. - Nous étudions les intégrales du type $\int_{0}^{t} \varphi(s) d \mathrm{~L}_{s}$, où $\varphi$ est une fonction borelienne positive localement bornée et où $\mathrm{L}_{t}$ est le temps local en 0 d'un processus de Bessel de dimension $\mathrm{d}, 0<\mathrm{d}<2$.

Titre En FRANÇAIS : Transformée d'Abel et intégrales du temps local de processus de Bessel

\section{INTRODUCTION}

Let $\left(X_{t}, t \geq 0\right)$ be a nice real-valued diffusion, with scale function $s$ and speed measure $m$; we are particularly interested in the case when $X_{t}$ is Brownian motion, or a Bessel process with dimension $\mathrm{d} \in] 0,2[$.

In a number of problems, the laws of inhomogeneous functionals

$$
\int_{0}^{t} f\left(s, X_{s}\right) d s
$$

\footnotetext{
${ }^{1}$ e-mail: Mihai.Gradinaru@iecn.u-nancy.fr
} 
are of interest (see, e.g. [3], [4], [23]). Such functionals may be represented as (inhomogeneous) integrals of the local times of $X$ :

$$
\int_{0}^{t} f\left(s, X_{s}\right) d s=\int_{0}^{\infty} m(d x) \int_{0}^{t} d_{s} \mathrm{~L}_{s}^{x} f(s, x)
$$

where

$$
\mathrm{L}_{t}^{x}=\lim _{\varepsilon \downarrow 0}(1 / m(x, x+\varepsilon)) \int_{0}^{t} d s \mathbb{1}_{\left\{x \leq X_{s} \leq x+\varepsilon\right\}}
$$

are the (diffusion) local times at level $x$ associated with $X$ (see, e.g. [11], p. 174).

Although the computations of the laws of $\int_{0}^{t} f\left(s, X_{s}\right) d s$ may be obtained, in theory, from the Feynman-Kac formula, these computations are not easy in practice. Thus, it seemed natural to first consider the "simplest" cases (in view of $(0.1)$ ), i.e. the computations of the laws of

$$
\mathrm{L}_{t}^{(\varphi)}:=\int_{0}^{t} \varphi(s) d \mathrm{~L}_{s}
$$

where $\mathrm{L}_{t}:=\mathrm{L}_{t}^{0}$ is the local time at level 0 .

Now, the moments of $\mathrm{L}_{t}^{(\varphi)}$ are easily obtained in terms of the densities $p_{t}(x, y)$ of the semi-group $P_{t}(x, d y)$ with respect to $m(d y)$, i.e.:

$$
P_{t}(x, d y)=p_{t}^{\cdot}(x, y) m(d y) .
$$

Indeed, it follows from (0.1) and the continuity of $p_{t}(x, y)$ (see, again, [11], p. 175), that:

$$
\mathrm{E}_{x}\left[d_{t} \mathrm{~L}_{t}^{y}\right]=p_{t}(x, y) d t .
$$

We denote, for simplicity, $q(t):=p_{t}(0,0)$. With the help of the Markov property, the moments of $\mathrm{L}_{t}^{(\varphi)}$ are given by:

$$
\begin{gathered}
\mathrm{E}_{0}\left[\left(\mathrm{~L}_{t}^{(\varphi)}\right)^{m}\right]=(m !) \mathrm{E}\left[\int_{0}^{t} \int_{s_{1}}^{t} \ldots \int_{s_{m-1}}^{t} d_{s_{1}} \mathrm{~L}_{s_{1}} \ldots d_{s_{m}} \mathrm{~L}_{s_{m}} \varphi\left(s_{1}\right) \ldots \varphi\left(s_{m}\right)\right] \\
=(m !) \int_{0}^{t} \int_{s_{1}}^{t} \ldots \int_{s_{m-1}}^{t} d s_{1} d s_{2} \ldots d s_{m} q\left(s_{1}\right) q\left(s_{2}-s_{1}\right) \ldots q\left(s_{m}-s_{m-1}\right) \\
\times \varphi\left(s_{1}\right) \ldots \varphi\left(s_{m}\right) .
\end{gathered}
$$

Using Fubini's theorem, we can write:

$$
\mathrm{E}_{0}\left[\left(\mathrm{~L}_{t}^{(\varphi)}\right)^{m}\right]=(m !) Q_{t}(\varphi Q \bullet(\varphi Q \bullet(\ldots Q \bullet(\varphi \mathbf{1}) \ldots))),
$$


where

$$
Q_{t}(\psi):=(Q \psi)(t)=\int_{0}^{t} q(t-s) \psi(s) d s
$$

In the particular case when $X$ is the Bessel process with dimension $\mathrm{d}=2(1+\mathrm{n})$, where $\mathrm{n} \in]-1,0[$, we get

$$
q(t)=k_{\mathrm{n}} t^{-\mathrm{n}-1}, k_{n}:=2^{-\mathrm{n}} \Gamma(\mathrm{n}+1)^{-1},
$$

so that $Q$ is then a multiple of the Abel integral operator (see section 1.5 below) with index $\alpha=-\mathrm{n}$. Hence, the Abel integral operators are naturally closely related to the laws of $\mathrm{L}_{t}^{(\varphi)}$ for Bessel processes (see also Remark 4.1).

More generally, the above computations, which could be extended to the computations of the moments of $\int_{0}^{t} f\left(s, X_{s}\right) d s$ are well-understood in the diffusion literature (see, e.g. [19] for some particular examples).

However, what may be a little newer is that in this paper is our characterization of the law of

$$
\mathrm{L}_{t}^{(\varphi)}(R):=\int_{0}^{t} \varphi(s) d \mathrm{~L}_{s}(R)
$$

for the Bessel process $R$, for the Bessel bridge, conditioned to take the value $y$ in time 1 , and in particular $y=0$. Recall that $\mathrm{L}_{1}^{(\mathbf{1})}(R)=\mathrm{L}_{1}(R)$ is a Mittag-Leffler random variable with parameter $|\mathrm{n}|$ (see, e.g. [7], p. 447 or [14], p. 129).

Moreover, we show that the random variables

$$
R_{Z} \text { and } \int_{0}^{Z} \varphi(1-Z+v) d \mathrm{~L}_{v}(R)
$$

are independent, and $\int_{0}^{Z} \varphi(1-Z+v) d \mathrm{~L}_{v}(R)$ is exponentially distributed with parameter 1 . Here, $Z$ is a random variable independent from $R$. Its probability density function, $\alpha_{f}$ on $[0,1]$, is the Abel transform of index $1+\mathrm{n}$ of the derivative of a regular increasing function $f$. The function $\varphi$ is related to $f$ by the equality $\varphi=\alpha_{f} / f$.

Clearly, the particular case $\mathrm{n}=-1 / 2$ corresponds to the process $|B|$, with $B$ the linear Brownian motion.

Our approach is based on two probabilistic representations for the solution of a partial differential equation with mixed boundary conditions. Using 
Abel's transform and these two representations (the backward Kolmogorov representation and the Fokker-Planck representation) we deduce some useful information on functionals of local time $\mathrm{L}_{t}^{(\varphi)}$. Some results obtained here may be proved using the dual predictable projection of the last passage time in 0 (see another proof of Corollary 3.4).

The plan of the paper is as follows. After some useful preliminaries on Bessel processes and Abel operators (section 1), we give the probabilistic representations for the solution of this partial differential equation and the analytic relations involving Abel's transform (section 2). In section 3 we state our main results. The proofs are given in section 4 . We also make a number of remarks. This paper is a complement and a generalization of [10]. One of the novelties in this paper is the importance of the Abel transform which we had not realized, hence also not introduced in our 1997 preprint. Finally, for practical and pedagogical, purposes, we collect in the Appendix the main formulas obtained in both papers.

\section{PRELIMINARIES}

In this section we review a few basic facts on Bessel processes and on Abel operators. For the proofs of these results, the reader may consult the book [17], Chap. XI (see, also, for a number of applications, [15], [20], [22]), respectively the book [9], Chap. 4.

\subsection{Bessel processes and Bessel semi-groups}

For any $\mathrm{d} \geq 0$ we denote by $R_{t}^{2}$ the square of a d-dimensional Bessel process, the unique solution of the stochastic differential equation

$$
R_{t}^{2}=R_{0}^{2}+2 \int_{0}^{t} \sqrt{R_{s}^{2}} d \beta_{s}+\mathrm{d} t
$$

where $\beta$ is a linear Brownian motion. The law of the square of a d-dimensional Bessel process, started at $x$, is denoted $Q_{x}^{(\mathrm{d})}$ and satisfies the following additivity property:

$$
Q_{x}^{(\mathrm{d})} * Q_{x^{\prime}}^{\left(\mathrm{d}^{\prime}\right)}=Q_{x+x^{\prime}}^{\left(\mathrm{d}+\mathrm{d}^{\prime}\right)},
$$

for every $\mathrm{d}, \mathrm{d}^{\prime}>0$ and $x, x^{\prime} \geq 0$. Take $0<\mathrm{d}<2$ and denote by $\mathrm{n}:=$ $\mathrm{d} / 2-1 \in]-1,0\left[\right.$ the index of the Bessel process, and $Q_{x}^{\mathrm{n}}:=Q_{x}^{(\mathrm{d})}$. From the additivity property we deduce the Laplace transform

$$
\mathrm{E}^{\mathrm{Q}_{x}^{\mathrm{n}}}\left[\exp \left(-\lambda R_{t}^{2}\right)\right]=(1+2 \lambda t)^{-(\mathrm{n}+1)} \exp (-\lambda x /(1+2 \lambda t)) .
$$


By inverting the Laplace transform we get, for $n>-1$, the density of the semi-group of the square of the Bessel process of index $\mathrm{n}$, started at $x$ :

$$
q_{t}^{\mathrm{n}}(x, y):=(2 t)^{-1}(y / x)^{\mathrm{n} / 2} \exp (-(x+y) / 2 t) \mathrm{I}_{\mathrm{n}}(\sqrt{x y} / t), t>0, x>0,
$$

where $\mathrm{I}_{\mathrm{n}}$ is the Bessel function of index $\mathrm{n}$. For $x=0$ this density becomes:

$$
q_{t}^{\mathrm{n}}(0, y):=(2 t)^{-\mathrm{n}-1} \Gamma(\mathrm{n}+1)^{-1} y^{\mathrm{n}} e^{-y / 2 t} .
$$

The square root of the square of a Bessel process of index $\mathrm{n}$, started at $a^{2}$, $R_{t}$, is called the Bessel process of index $\mathrm{n}$ started at $a \geq 0$. Its law will be denoted by $\mathrm{P}_{a}^{\mathrm{n}}$. The density of the semi-group is obtained from that of the square of a Bessel process of index $n$, by a straightforward change of variable. We obtain, for $\mathrm{n}>-1$,

$$
p_{t}^{\mathrm{n}}(x, y):=(y / t)(y / x)^{\mathrm{n}} \exp \left(-\left(x^{2}+y^{2}\right) / 2 t\right) \mathrm{I}_{\mathrm{n}}(x y / t), t, x>0,
$$

and

$$
p_{t}^{\mathrm{n}}(0, y):=2^{-\mathrm{n}} \Gamma(\mathrm{n}+1)^{-1} t^{-\mathrm{n}-1} y^{2 \mathrm{n}+1} e^{-y^{2} / 2 t}, y>0 .
$$

From (1.1) we see that, for positive Borel functions $u, v$,

$$
<\Pi_{t}^{\mathrm{n}} u, v>_{\nu}=<u, \Pi_{t}^{\mathrm{n}} v>_{\nu}, t>0
$$

where $\Pi_{t}^{\mathrm{n}}$ denote the semi-group of the Bessel process of index $\mathrm{n}$

$$
\Pi_{t}^{\mathrm{n}} u(a):=\mathrm{E}_{a}^{\mathrm{n}}\left[u\left(R_{t}\right)\right],
$$

and

$$
<u, v>_{\nu}:=\int_{0}^{\infty} u(x) v(x) x^{2 \mathrm{n}+1} d x .
$$

Hence the semi-group is symmetric with respect to the invariant measure given by:

$$
\nu(d x):=x^{2 \mathrm{n}+1} \mathbb{1}_{0, \infty[}(x) d x
$$

The infinitesimal generator of the Bessel process is

$$
\mathcal{L}=(1 / 2)\left(\partial^{2} / \partial x^{2}\right)+((2 \mathrm{n}+1) / 2 x)(\partial / \partial x)
$$


On

$$
\mathcal{D}(\mathcal{L}):=\left\{f: f, \mathcal{L} f \in C_{b}\left(\left[0, \infty[), \lim _{x \downarrow 0} x^{2 \mathrm{n}+1} f^{\prime}(x)=0\right\}\right.\right.
$$

(see also [13], p. 761, or [6], pp. 114-115).

A continuous process $\left(r_{t}: t \in\left[0, t_{0}\right]\right)$, the law of which is equal to

$$
\mathrm{P}_{a, b}^{\mathrm{n}, t_{0}}(\cdot):=\mathrm{P}_{a}^{\mathrm{n}}\left(\cdot \mid R_{t_{0}}=b\right)
$$

is called the Bessel bridge from $a$ to $b$ over $\left[0, t_{0}\right]$. In the sequel, we shall be interested in the Bessel bridge $r_{t}$ from 0 to 0 over $[0,1-s]$, with $s \in[0,1]$.

\subsection{Absolute continuity properties and law of the first hitting time of 0}

It is known that for $-1<\mathrm{n}<0$ the point 0 is reached a.s. under $\mathrm{P}_{x}^{\mathrm{n}}$ and it is instantaneously reflecting. Let us denote $T_{0}:=\inf \left\{t>0: R_{t}=0\right\}$ and $\mathcal{F}_{t}:=\sigma\left\{R_{s}: s \leq t\right\}$. Then, the laws of the Bessel processes satisfy the following absolute continuity property:

$$
\left(\mathrm{P}_{a}^{\mathrm{n}}\right)_{\mid \mathcal{F}_{t} \cap\left\{t<T_{0}\right\}}=\left(R_{t} / a\right)^{\mathrm{n}} \exp \left(-\left(\mathrm{n}^{2} / 2\right) \int_{0}^{t} d s / R_{s}^{2}\right) \cdot\left(\mathrm{P}_{a}^{0}\right)_{\mid \mathcal{F}_{t}},
$$

an equality which is satisfied for every $\mathrm{n} \geq-1$. Note that $\mathrm{P}_{a}^{0}$ is the law of the 2-dimensional Bessel process starting from $a$. From this we deduce, for $-1 \leq \mathrm{n}<0$,

$$
\left(\mathrm{P}_{a}^{\mathrm{n}}\right)_{\mid \mathcal{F}_{t} \cap\left\{t<T_{0}\right\}}=\left(a / R_{t}\right)^{2|\mathrm{n}|} \cdot\left(\mathrm{P}_{a}^{|\mathrm{n}|}\right)_{\mid \mathcal{F}_{t}},
$$

$(|\mathrm{n}|=-\mathrm{n}$, since $-1 \leq \mathrm{n}<0)$.

Let us denote by $\bar{\Pi}_{t}^{\mathrm{n}}$ the semi-group of the Bessel process killed at $T_{0}$

$$
\bar{\Pi}_{t}^{\mathrm{n}} u(x):=\mathrm{E}_{x}^{\mathrm{n}}\left[u\left(R_{t}\right) \mathbb{I}_{\left\{t<T_{0}\right\}}\right],
$$

and we can deduce from (1.2) and (1.4), that, for positive Borel functions $u, v$,

$$
<\bar{\Pi}_{t}^{\mathrm{n}} u, v>_{\nu}=<u, \bar{\Pi}_{t}^{\mathrm{n}} v>_{\nu}, t>0 .
$$

Moreover, by (1.4), we can deduce the Williams' time-reversal property: the processes 


$$
\left\{R_{T_{0}-t}: t<T_{0}\right\} \text { under } \mathrm{P}_{x}^{\mathrm{n}} \text { and }\left\{R_{t}: t<\gamma_{x}\right\} \text { under } \mathrm{P}_{0}^{|\mathrm{n}|}
$$

are identical in law (cf. [18], p. 1158 or [15], p. 302). Here we denote $\gamma_{x}:=\sup \left\{t: R_{t}=x\right\}$. Therefore

$$
\mathrm{P}_{x}^{\mathrm{n}}\left(T_{0} \in d t\right)=\mathrm{P}_{0}^{|\mathrm{n}|}\left(\gamma_{x} \in d t\right) .
$$

Using a result in [15], p. 329, (or [17], p. 308, Ex. $(4.16), 5^{\circ}$ ), we can write

$$
P_{0}^{|\mathrm{n}|}\left(\gamma_{x} \in d t\right)=(|\mathrm{n}| / x) p_{t}^{|\mathrm{n}|}(0, x) d t .
$$

Using (1.1') we obtain the density of $T_{0}$, under $\mathrm{P}_{x}^{\mathrm{n}}$,

$$
\phi(x, t):=(d / d t) \mathrm{P}_{x}^{\mathrm{n}}\left(T_{0} \leq t\right)=2^{\mathrm{n}} \Gamma(|\mathrm{n}|)^{-1} t^{\mathrm{n}-1} x^{-2 \mathrm{n}} e^{-x^{2} / 2 t}, x, t>0
$$

(for this particular result and $\mathrm{n} \geq 1 / 2$, see also [8], p. 864).

We also note that, using the strong Markov property at $T_{0}$ we get, for $y>0$,

$$
p_{t}^{\mathrm{n}, \nu}(0, y)=p_{t}^{\mathrm{n}, \nu}(y, 0)=\int_{0}^{t} \phi(y, s) p_{t-s}^{\mathrm{n}, \nu}(0,0) d s .
$$

Here $p_{t}^{\mathrm{n}, \nu}(0, y)$ is the density of the semi-group $\Pi_{t}^{\mathrm{n}}$ with respect to the invariant measure $\nu$.

\subsection{Local time at level 0}

The local time at level $x$ for the Bessel process $L_{t}^{x}(R)$ can be defined as an occupation density: for every positive Borel function $h$,

$$
\int_{0}^{t} h\left(R_{s}\right) d s=2 \int_{0}^{\infty} h(x) \mathrm{L}_{t}^{x}(R) x^{2 \mathrm{n}+1} d x .
$$

Note that we use a different normalisation than in the formula in [17], p. 308, Ex. (4.16), $4^{\circ}$ and also than in (0.1) in the Introduction.

This choice of local times agrees with the fact that $R_{t}^{2|\mathrm{n}|}-2|\mathrm{n}| \mathrm{L}_{t}^{0}(R)$ is a martingale. Indeed, $R_{t}^{2|\mathrm{n}|}$ is a $\mathcal{F}_{t}$-submartingale and it suffices to write its Doob-Meyer decomposition to obtain $\mathrm{L}_{t}^{0}(R)$ (up to a constant factor). We often denote $\mathrm{L}_{t}(R)$ instead of $\mathrm{L}_{t}^{0}(R)$.

Let us now mention two related scaling properties. Firstly, the Bessel process has the Brownian scaling property, that is, for any real $c>0$, 
the processes $R_{c t}$ and $c^{1 / 2} R_{t}$ have the same law, when $R_{0}=0$. Secondly, $\left\{\mathrm{L}_{t}(R): t \geq 0\right\}$ inherits from $R$ the following scaling property: indeed, by (1.9),

$$
\left\{\mathrm{L}_{c t}(R): t \geq 0\right\} \stackrel{(\text { law })}{=}\left\{c^{|\mathrm{n}|} \mathrm{L}_{t}(R): t \geq 0\right\}
$$

We also recall that,

$$
\int_{0}^{1} \varphi(t) d \mathrm{~L}_{t}(R)<\infty \Leftrightarrow \int_{0}^{1} \varphi(t) t^{-\mathrm{n}-1} d t<\infty
$$

(see [16], p. 655 and [12], p. 44 for the case of Brownian motion, $\mathrm{n}=-1 / 2$ ).

\subsection{A simple path decomposition and the Bessel meander}

It is known that, conditionally on $g_{t},\left\{R_{s}: s \leq g_{t}\right\}$ is independent of $\left\{R_{s}: s \geq g_{t}\right\}$, where $g_{t}:=\sup \left\{s \leq t: R_{s}=0\right\}$. More precisely, the Bessel meander

$$
m_{t}(u):=\left(1 / \sqrt{t-g_{t}}\right) R_{g_{t}+u\left(t-g_{t}\right)}, u \in[0,1]
$$

is independent of $\mathcal{F}_{g_{t}}$ (see [20], p. 42). Let us recall also that, for fixed $t>0$

$$
g_{t} \stackrel{(\text { law })}{=} t Z_{-\mathrm{n}, 1+\mathrm{n}}
$$

where by $Z_{a, b}$ we denote a beta random variable with parameters $a, b>0$ :

$$
\mathrm{P}\left(Z_{a, b} \in d u\right)=\mathrm{B}(a, b)^{-1} u^{a-1}(1-u)^{b-1} \mathbb{I}_{] 0,1[}(u) d u .
$$

Moreover, for every $t>0$,

$$
m_{t}(1)=R_{t} / \sqrt{t-g_{t}} \stackrel{(\text { law })}{=} \sqrt{2 \mathcal{E}(1)}
$$

where $\mathcal{E}(1)$ denotes an exponential variable with parameter 1 and $\sqrt{2 \mathcal{E}(1)}$ is a Rayleigh random variable having density $u e^{-u^{2} / 2} \mathbb{1}_{[0, \infty[}(u)$.

\subsection{Abel's integral operator}

The Abel transform of a positive Borel function $f$ on $[0, \infty[$, is defined as 


$$
J^{\alpha} f(t):=\Gamma(\alpha)^{-1} \int_{0}^{t}(t-u)^{\alpha-1} f(u) d u, t \geq 0,
$$

where $\alpha>0$. The operator $J^{\alpha}$ is called Abel integral operator, or fractional integral operator. Our principal interest is for $0<\alpha \leq 1$. Clearly, $J^{1}$ is the ordinary integration operator, that is $J^{1} f$ is the primitive of $f$.

By straightforward calculation we can verify that, for $\alpha, \beta>0$,

$$
J^{\alpha} \circ J^{\beta}=J^{\alpha+\beta} .
$$

This, together with the previous remark, implies, if $f(0)=0$,

$$
J^{\alpha+1}\left(f^{\prime}\right)=J^{\alpha} f
$$

Let us also note that if $\epsilon_{\gamma}(t):=t^{\gamma}, \gamma>-1$, then

$$
J^{\alpha} \epsilon_{\gamma}=(\Gamma(\gamma+1) / \Gamma(\alpha+\gamma+1)) \epsilon_{\alpha+\gamma}
$$

\section{TWO PROBABILISTIC REPRESENTATIONS}

Let us consider $\omega_{0}$ a continuous function with growth less than exponential at infinity, and $f$ a continuous function such that $f \in \mathrm{C}^{1}(] 0, \infty[)$. Assume that $\omega_{0}(0)=f(0)=0$.

\subsection{Dirichlet and Fokker-Planck representations}

Recall that $\mathcal{L}$ is the generator of the Bessel process (see (1.3)). There is existence and uniqueness of the solution of the problem

$$
(\partial \omega / \partial t)(t, x)=(\mathcal{L} \omega)(t, x), t, x>0
$$

with

$$
\omega(0, x)=\omega_{0}(x), x \geq 0,
$$

and

$$
\omega(t, 0)=f(t), t \geq 0 .
$$


It admits both a Dirichlet representation (Proposition 2.1 below) and a Fokker-Planck representation (Proposition 2.2 below). We shall then compare these two representations (Proposition 2.3 below).

Proposition 2.1. - The solution $\omega(t, x)$ of (2.1)-(2.3) can be written as

$$
\omega(t, x)=\omega_{1}(t, x)+\omega_{2}(t, x), t, x \geq 0,
$$

where

$$
\begin{aligned}
\omega_{1}(t, x):=\mathrm{E}_{x}^{\mathrm{n}}\left[\omega_{0}\left(R_{t}\right) \mathbb{I}_{\left\{t<T_{0}\right\}}\right], t, x \geq 0, \\
\omega_{2}(t, x):=\mathrm{E}_{x}^{\mathrm{n}}\left[f\left(t-T_{0}\right) \mathbb{I}_{\left\{t \geq T_{0}\right\}}\right] \\
=\int_{0}^{t} \phi(x, s) f(t-s) d s, t \geq 0, x>0 .
\end{aligned}
$$

Proof. - To obtain (2.4) it suffices to solve the Dirichlet problem for the operator $\mathcal{L}-\partial / \partial t$ on $[0, \infty[\times[0, t]$ (see also [10], Proposition 1.2).

Proposition 2.2. - The solution $\omega(t, x)$ of (2.1)-(2.3) (also) admits the following Fokker-Planck representation

$$
\begin{gathered}
y^{2 \mathrm{n}+1} \omega(t, y)=\int_{0}^{\infty} \omega_{0}(x) p_{t}^{\mathrm{n}}(x, y) x^{2 \mathrm{n}+1} d x \\
\times \mathrm{E}_{x}^{\mathrm{n}}\left[\exp \left(-\int_{0}^{t} \varphi(s) d \mathrm{~L}_{s}(R)\right) \mid R_{t}=y\right], t, y \geq 0,
\end{gathered}
$$

where, if $\omega$ is given by $(2.4)$,

$$
\varphi(t):=(1 / f(t)) \lim _{x \downarrow 0} x^{2 \mathrm{n}+1}(\partial \omega / \partial x)(t, x), t \geq 0,
$$

REMARK. - (i) Unlike formulae (2.5) and (2.6), formula (2.7) features the "unknown" function $\omega$ both on its left and right hand sides.

(ii) The decomposition (2.4), $\omega=\omega_{1}+\omega_{2}$ yields a corresponding decomposition $\varphi=\varphi_{1}+\varphi_{2}$ (with obvious notation). In Proposition 2.4 below, $f \varphi_{1}$ and $f \varphi_{2}$ shall be given in a closed form, in terms respectively of $\omega_{0}$ and $f$.

Proof of Proposition 2.2. - Let $\varphi$ be given by (2.8) and define

$$
M_{t}:=\exp \left(-\int_{0}^{t} \varphi(s) d \mathrm{~L}_{s}(R)\right) .
$$


We introduce, for $t \geq 0, x \geq 0$, the function $\tilde{\omega}(t, x)$ defined by

$$
<h(\cdot), \tilde{\omega}(t, \cdot)>_{\nu}:=\mathrm{E}_{\omega_{0} \nu}^{\mathrm{n}}\left[h\left(R_{t}\right) M_{t}\right]
$$

Here $h$ is an arbitrary bounded positive Borel function and we denote

$$
\mathrm{P}_{\omega_{0} \nu}^{\mathrm{n}}(A):=\int_{0}^{\infty} \mathrm{P}_{x}^{\mathrm{n}}(A) \omega_{0}(x) x^{2 \mathrm{n}+1} d x .
$$

i) Let $g:[0, \infty[\times[0, \infty[\rightarrow[0, \infty[$ be a smooth function with compact support disjoint of $[0, \infty[\times\{0\}$. Ito's formula gives

$$
\begin{gathered}
g\left(t, R_{t}\right) M_{t}=g(0, x)+\int_{0}^{t}(\partial g / \partial x)\left(s, R_{s}\right) M_{s} d B_{s} \\
+\int_{0}^{t}(\partial g / \partial s+\mathcal{L} g)\left(s, R_{s}\right) M_{s} d s
\end{gathered}
$$

so, taking the expectation, we can write

$$
\begin{gathered}
<g(t, \cdot), \tilde{\omega}(t, \cdot)>_{\nu}=<g(0, \cdot), \omega_{0}(\cdot)>_{\nu} \\
+\int_{0}^{t}<(\partial g / \partial s+\mathcal{L} g)(s, \cdot), \tilde{\omega}(s, \cdot)>_{\nu} d s
\end{gathered}
$$

Taking the derivative with respect to $t$ we get

$$
<g(t, \cdot),(\partial \tilde{\omega} / \partial t)(t, \cdot)>_{\nu}=<(\mathcal{L} g)(t, \cdot), \tilde{\omega}(t, \cdot)>_{\nu}
$$

Since the support of $g$ is disjoint of $[0, \infty[\times\{0\}$, integrating by parts we deduce

$$
<g(t, \cdot),(\partial \tilde{\omega} / \partial t)(t, \cdot)>_{\nu}=<g(t, \cdot),(\mathcal{L} \tilde{\omega})(t, \cdot)>_{\nu},
$$

Hence $\tilde{\omega}$ satisfies $(2.1)$.

ii) Let us consider $u$ and $v$ two smooth functions defined on $[0, \infty[$ and take now $g:[0, \infty[\times[0, \infty[\rightarrow \mathbb{R}$ a smooth function with compact support such that $g(t, x):=u(t) x^{2|\mathrm{n}|}+v(t)$ in a neighbourhood of $[0, \infty[\times\{0\}$. Since $R_{t}^{2|\mathrm{n}|}-2|\mathrm{n}| \mathrm{L}_{t}(R)$ is a martingale, Ito's formula gives

$$
\begin{gathered}
g\left(t, R_{t}\right) M_{t}=g(0, x)+\int_{0}^{t}(\partial g / \partial x)\left(s, R_{s}\right) M_{s} d B_{s}+\int_{0}^{t} \mathcal{L} g\left(s, R_{s}\right) M_{s} d s \\
\quad+\int_{0}^{t}(\partial g / \partial s)\left(s, R_{s}\right) M_{s} d s+\int_{0}^{t}(2|\mathrm{n}| u(s)-\varphi(s) v(s)) M_{s} d \mathrm{~L}_{s}(R) .
\end{gathered}
$$


iii) Assume that $u, v, \varphi$ verify

$$
2|\mathrm{n}| u(s)-\varphi(s) v(s)=0 .
$$

By the same arguments of i) and by (c), we obtain again (b). Since the assumptions on the function $g$ are different, let us detail the integration by parts on the right hand of (b). Using the form (1.3) of the operator $\mathcal{L}$ we can write,

$$
\begin{gathered}
<(\mathcal{L} g)(t, \cdot), \tilde{\omega}(t, \cdot)>_{\nu} \\
=\left(\frac{1}{2} \frac{\partial g}{\partial x}(t, x) x^{2 \mathrm{n}+1} \tilde{\omega}(t, x)\right)_{x \downarrow 0}^{x \uparrow \infty}-\frac{1}{2} \int_{0}^{\infty} \frac{\partial g}{\partial x}(t, x) x^{2 \mathrm{n}+1} \frac{\partial \tilde{\omega}}{\partial x}(t, x) d x \\
=\left(\frac{1}{2} \frac{\partial g}{\partial x}(t, x) x^{2 \mathrm{n}+1} \tilde{\omega}(t, x)\right)_{x \downarrow 0}-\left(\frac{1}{2} g(t, x) x^{2 \mathrm{n}+1} \frac{\partial \tilde{\omega}}{\partial x}(t, x)\right)_{x \downarrow 0}^{x \uparrow \infty} \\
+\frac{1}{2} \int_{0}^{\infty} g(t, x) \frac{\partial}{\partial x}\left(x^{2 \mathrm{n}+1} \frac{\partial \tilde{\omega}}{\partial x}(t, x)\right) d x \\
\left.=|\mathrm{n}| u(t) \tilde{\omega}(t, 0)-\frac{v(t)}{2} \lim _{x \downarrow 0} x^{2 \mathrm{n}+1} \frac{\partial \tilde{\omega}}{\partial x}(t, x)+<g(t, \cdot),(\mathcal{L} \tilde{\omega})(t, \cdot)\right\rangle_{\nu} .
\end{gathered}
$$

Since $\tilde{\omega}$ verifies (2.1), replacing the above equality in (b), we deduce

$$
2|\mathrm{n}| u(t) \tilde{\omega}(t, 0)-v(t) \lim _{x \downarrow 0} x^{2 \mathrm{n}+1}(\partial \tilde{\omega} / \partial x)(t, x)=0 .
$$

iv) Since $u, v$ are arbitrary functions, combining (c) and (d) we get

$$
\lim _{x \downarrow 0} x^{2 \mathrm{n}+1} \frac{\partial \omega}{\partial x}(t, x)-\frac{\omega(t, 0)}{\tilde{\omega}(t, 0)} \lim _{x \downarrow 0} x^{2 \mathrm{n}+1} \frac{\partial \tilde{\omega}}{\partial x}(t, x)=0 .
$$

Moreover, $\tilde{\omega}$ satisfies

$$
\tilde{\omega}(0, x)=\omega_{0}(x), \forall x \geq 0 .
$$

Since $\tilde{\omega}$ and $\omega$ verify the same parabolic equation (2.1) with the same initial and mixed boundary conditions $(2.2)$ and (2.3), we deduce that $\tilde{\omega}=\omega$.

$v)$ Therefore, by (a) we deduce that, for every bounded positive Borel function $h$,

$$
\begin{gathered}
\int_{0}^{\infty} h(y) \omega(t, y) y^{2 \mathrm{n}+1} d y=\int_{0}^{\infty} \omega_{0}(x) x^{2 \mathrm{n}+1} d x \\
\times \mathrm{E}_{x}^{\mathrm{n}}\left[h\left(R_{t}\right) \exp \left(-\int_{0}^{t} \varphi(s) d \mathrm{~L}_{s}(R)\right)\right] .
\end{gathered}
$$


By conditioning with respect to $R_{t}=y$ on the right hand side of the above equality we obtain (2.7), since $h$ is arbitrary.

Now, the Dirichlet representation of $\omega$ naturally decomposes $\omega$ into $\omega_{1}+\omega_{2}$ and, in the next proposition, we also find this decomposed form in the Fokker-Planck representation.

Proposition 2.3. - The decomposition $\omega=\omega_{1}+\omega_{2}$ appears as follows in the Fokker-Planck representation: for $t, y \geq 0$,

$$
\begin{aligned}
y^{2 \mathrm{n}+1} \omega_{1}(t, y)= & \int_{0}^{\infty} \omega_{0}(x) p_{t}^{\mathrm{n}}(x, y) x^{2 \mathrm{n}+1} d x \mathrm{P}_{x}^{\mathrm{n}}\left[T_{0}>t \mid R_{t}=y\right] \\
& =y^{2 \mathrm{n}} \int_{0}^{\infty} \omega_{0}(x) p_{t}^{|\mathrm{n}|}(x, y) x d x
\end{aligned}
$$

and

$$
\begin{gathered}
y^{2 \mathrm{n}+1} \omega_{2}(t, y)=\int_{0}^{\infty} \omega_{0}(x) p_{t}^{\mathrm{n}}(x, y) x^{2 \mathrm{n}+1} d x \\
\times \mathrm{E}_{x}^{\mathrm{n}}\left[\exp \left(-\int_{0}^{t} \varphi(s) d \mathrm{~L}_{s}(R)\right) \mathbb{1}_{\left\{t \geq T_{0}\right\}} \mid R_{t}=y\right],
\end{gathered}
$$

or, equivalently,

$$
\begin{gathered}
y^{2 \mathrm{n}+1} \omega_{2}(t, y)=\int_{0}^{t} d s \mathrm{E}_{0}^{\mathrm{n}}\left[\exp \left(-\int_{0}^{t-s} \varphi(s+v) d \mathrm{~L}_{v}(R)\right) \mid R_{t-s}=y\right] \\
\times \int_{0}^{\infty} x^{2 \mathrm{n}+1} \omega_{0}(x) \phi(x, s) p_{t-s}^{\mathrm{n}}(0, y) d x, t, y \geq 0 .
\end{gathered}
$$

Here, $p_{t}^{\mathrm{n}}$ is given by (1.1), (1.1'), $\phi$ by (1.7) and $\varphi$ by (2.8).

Proof. - The first equality in (2.9) is only the translation of the symmetry of the semi-group of the killed Bessel process $\bar{\Pi}_{t}^{\mathrm{n}}$, with respect to the measure $\nu$ (see (1.5)). The second equality in (2.9) is obtained thanks to formulae (1.1) and (1.4), from which the formula

$$
\mathrm{P}_{x}^{\mathrm{n}}\left(T_{0}>t \mid R_{t}=y\right)=\left(\mathrm{I}_{|\mathrm{n}|} / \mathrm{I}_{\mathrm{n}}\right)(x y / t)
$$

follows. To get (2.11) we use the strong Markov property at $T_{0}$.

\subsection{Analytic relations involving Abel's transform}

There exist some analytic relations between the functions $\omega_{0}, f$ and $\varphi$ involving Abel's transform (cf. Proposition 2.4 below). 
Recall that $f$ is a continuous function such that $f \in C^{1}(] 0, \infty[)$ and $f(0)=0$. Let us introduce, for $t \geq 0$, and $-1<\mathrm{n}<0$, the constant

$$
c_{\mathrm{n}}:=2^{\mathrm{n}+1} \Gamma(\mathrm{n}+1) / \Gamma(|\mathrm{n}|),
$$

and the function

$$
\alpha_{f}(t):=c_{\mathrm{n}}\left(J^{\mathrm{n}+1} f^{\prime}\right)(t) .
$$

Recall that, from (1.14'), we have $\left(J^{1} \alpha_{f}\right)(t)=c_{\mathrm{n}}\left(J^{\mathrm{n}+1} f\right)(t)$, hence

$$
k_{f}:=\int_{0}^{1} \alpha_{f}(t) d t=c_{\mathrm{n}}\left(J^{\mathrm{n}+1} f\right)(1) .
$$

We now give the promised explicit representations of $\varphi_{1}$ and $\varphi_{2}$ (see the remark following Proposition 2.2):

Proposition 2.4. - Assume that $\varphi$ is given by (2.8) with $\omega$ the solution of (2.1)-(2.3). Write $\varphi=\varphi_{1}+\varphi_{2}$, where

$$
\varphi_{i}(t):=(1 / f(t)) \lim _{x \downarrow 0} x^{2 \mathrm{n}+1}\left(\partial \omega_{i} / \partial x\right)(t, x), i=1,2 .
$$

Then, we have

$$
\begin{gathered}
\left(\varphi_{1} f\right)(t)=\left(2^{\mathrm{n}+1} / \Gamma(|\mathrm{n}|)\right) t^{\mathrm{n}-1} \int_{0}^{\infty} y \omega_{0}(y) e^{-y^{2} / 2 t} d y, t>0, \\
\left(\varphi_{2} f\right)(t)=-\alpha_{f}(t), t>0
\end{gathered}
$$

and, consequently:

$$
\left(\alpha_{f}+\varphi f\right)(t)=\left(2^{\mathrm{n}+1} / \Gamma(|\mathrm{n}|)\right) t^{\mathrm{n}-1} \int_{0}^{\infty} y \omega_{0}(y) e^{-y^{2} / 2 t} d y, t>0 .
$$

Proof. We need to compute the limits in (2.8'). First, using (2.9) and (1.1), we can write

$$
\omega_{1}(t, x)=\left(1 /\left(t x^{n}\right)\right) \int_{0}^{\infty} y^{\mathrm{n}+1} \omega_{0}(y) \exp \left(-\left(x^{2}+y^{2}\right) / 2 t\right) \mathrm{I}_{|\mathrm{n}|}(x y / t) d y
$$

Since

$$
\mathrm{I}_{|\mathrm{n}|}(y) \sim \frac{(y / 2)^{|\mathrm{n}|}}{\Gamma(|\mathrm{n}|+1)}, \mathrm{I}_{|\mathrm{n}|}^{\prime}(y) \sim \frac{(y / 2)^{|\mathrm{n}|-1}}{2 \Gamma(|\mathrm{n}|)}, \text { as } y \sim 0 .
$$


(2.14) follows from (a) by direct calculation.

On the other hand, by (2.6) and (1.7), we can write

$$
\begin{gathered}
x^{2 \mathrm{n}}\left(\omega_{2}(t, x)-\omega_{2}(t, 0)\right)=\left(2^{\mathrm{n}} / \Gamma(|\mathrm{n}|)\right) \\
\times\left(\int_{0}^{t} s^{\mathrm{n}-1} e^{-x^{2} / 2 s}(f(t-s)-f(t)) d s-f(t) \int_{t}^{\infty} s^{\mathrm{n}-1} e^{-x^{2} / 2 s} d s\right),
\end{gathered}
$$

from which we deduce

(b) $(2|\mathrm{n}|)^{-1}\left(\varphi_{2} f\right)(t)=\left(2^{\mathrm{n}} / \Gamma(|\mathrm{n}|)\right)\left(\int_{0}^{t} s^{\mathrm{n}-1}(f(t-s)-f(t)) d s+t^{\mathrm{n}} f(t) / \mathrm{n}\right)$.

To obtain (2.15), we write in (b), $f(t-s)-f(t)=\int_{t}^{t-s} f^{\prime}(u) d u$, and, by straighforward calculation, we find $(2.12$ ').

\section{MAIN RESULTS}

Before stating our new results, we explain the guiding idea behind them: suppose $\omega_{0}$ and $f$ are given. We shall write another form of (2.11), using (2.6) and (2.16). For $t=1$ and every $y \geq 0$, we get

$$
\begin{gathered}
y \int_{0}^{1}\left(2^{\mathrm{n}+1} / \Gamma(|\mathrm{n}|)\right) s^{\mathrm{n}-1} e^{-y^{2} / 2 s} f(1-s) d s \\
=\int_{0}^{1}\left(\alpha_{f}+\varphi f\right)(s) p_{1-s}^{\mathrm{n}}(0, y) d s \\
\times \mathrm{E}_{0}^{\mathrm{n}}\left[\exp \left(-\int_{0}^{1-s} \varphi(s+v) d \mathrm{~L}_{v}(R)\right) \mid R_{1-s}=y\right],
\end{gathered}
$$

since, by (1.7),

$$
x^{2 \mathrm{n}+1} \omega_{0}(x) \phi(x, s)=2^{\mathrm{n}} \Gamma(|\mathrm{n}|)^{-1} x \omega_{0}(x) s^{\mathrm{n}-1} e^{-x^{2} / 2 s}
$$

The first above equality was obtained using the two probabilistic representations (2.1)-(2.3) and Abel's transform. We see that the given function $\omega_{0}$ does not appear in the above equality. It suggests the following general result, where, this time $f$ and $\varphi$ are given as new parameters:

Theorem 3.1. - Consider the continuous functions $f:[0,1] \rightarrow[0, \infty[$ and $\varphi:[0,1] \rightarrow\left[0, \infty\left[\right.\right.$. Assume that $\left.\left.f \in C^{1}(] 0,1\right]\right), f(0)=0$ and that $t^{|\mathrm{n}|} \varphi(t)$ converges as $t \downarrow 0$. If $\alpha_{f}$ is given by (2.12'), then, for every $y \geq 0$, 


$$
\begin{gathered}
\int_{0}^{1}\left(\alpha_{f}+\varphi f\right)(1-s) p_{s}^{\mathrm{n}}(0, y) d s \\
\times \mathrm{E}_{0}^{\mathrm{n}}\left[\exp \left(-\int_{0}^{s} \varphi(1-s+v) d \mathrm{~L}_{v}(R)\right) \mid R_{s}=y\right] \\
=\left(2^{\mathrm{n}+1} / \Gamma(|\mathrm{n}|)\right) y \int_{0}^{1} s^{\mathrm{n}-1} e^{-y^{2} / 2 s} f(1-s) d s
\end{gathered}
$$

or, equivalently, for any positive bounded Borel function $h$,

$$
\begin{gathered}
\int_{0}^{1}\left(\alpha_{f}+\varphi f\right)(1-s) d s \\
\times \mathrm{E}_{0}^{\mathrm{n}}\left[h\left(R_{s}\right) \exp \left(-\int_{0}^{s} \varphi(1-s+v) d \mathrm{~L}_{v}(R)\right)\right] \\
=\left(2^{\mathrm{n}+1} / \Gamma(|\mathrm{n}|)\right) \int_{0}^{\infty} y h(y) d y \int_{0}^{1} s^{\mathrm{n}-1} e^{-y^{2} / 2 s} f(1-s) d s .
\end{gathered}
$$

In Remark 4.13 we prove a reciprocal result to Theorem 3.1. As consequences of this theorem, we can prove the following interesting results: Propositions 3.2 and 3.3 below concern the Bessel process, whereas Propositions 3.5 and 3.6 below concern the Bessel bridge $r$ (see also the end of the section 1.1).

Proposition 3.2. - Consider a continuous increasing function $f:[0,1] \rightarrow$ $\left[0, \infty\left[\right.\right.$ such that $\left.\left.f \in C^{1}(] 0,1\right]\right), f(0)=0$ and $f^{\prime}(t) \sim c t^{p}$ (for some constants $c, p>0)$ as $t \downarrow 0$. We also assume, without loss of generality, that the function $f$ is chosen such that the constant $k_{f}$ in (2.13) equals 1 . Consider a random variable $Z$ with the probability density function $\alpha_{f}(1-s) \mathbb{I}_{[0,1]}(s)$, independent of $R$. Then, for $\varphi:=\alpha_{f} / f$, the random variables

$$
R_{Z} \text { and } \int_{0}^{Z} \varphi(1-Z+v) d \mathrm{~L}_{v}(R)
$$

are independent. Moreover,

$$
\int_{0}^{Z} \varphi(1-Z+v) d \mathrm{~L}_{v}(R) \stackrel{(\mathrm{law})}{=} \mathcal{E}(1)
$$

Proposition 3.3. - Consider a continuous function $\varphi:] 0,1] \rightarrow[0, \infty[$ such that $t^{|\mathrm{n}|} \varphi(t)$ converges as $t \downarrow 0$. For $\lambda>0$, we denote 


$$
\Lambda_{\varphi}(a):=\mathrm{E}_{0}^{\mathrm{n}}\left[\exp \left(-\lambda \int_{0}^{1-a} \varphi(a+v) d \mathrm{~L}_{v}(R)\right)\right], a \in[0,1] .
$$

Then,

$$
\left(I+\left(\lambda / c_{\mathrm{n}}\right) \mathcal{A}_{\varphi}\right) \Lambda_{\varphi}=\mathbf{1}
$$

where, $c_{\mathrm{n}}$ is given by (2.12), and we denote, for a positive Borel function $h$,

$$
\left(\mathcal{A}_{\varphi} h\right)(a):=J^{-\mathrm{n}}(\tilde{\varphi} \tilde{h})(1-a), a \in[0,1] .
$$

Here and elsewhere $\tilde{h}(a):=h(1-a)$. Hence,

$$
\Lambda_{\varphi}(a)=\sum_{k \geq 0}\left(-\lambda / c_{\mathrm{n}}\right)^{k}\left(\mathcal{A}_{\varphi}^{k} \mathbf{1}\right)(a), a \in[0,1]
$$

Corollary 3.4. - For any random variable $Z>0$ a.s., independent of $R$, the integral

$$
\left(2^{\mathrm{n}+1} / \Gamma(|\mathrm{n}|)\right) \int_{0}^{Z}(Z-v)^{\mathrm{n}} d \mathrm{~L}_{v}(R)
$$

is a standard exponential variable and is independent of $Z$.

In Remark 4.6 we prove a reciprocal result to Corollary 3.4.

Proposition 3.5. - Consider a continuous increasing function $f:[0,1] \rightarrow$ $\left[0, \infty\left[\right.\right.$ such that $\left.\left.f \in C^{1}(] 0,1\right]\right), f(0)=0$ and $f^{\prime}(t) \sim c t^{p}$ (for some constants $c, p>0)$ as $t \downarrow 0$. We also assume, without loss of generality, that the function $f$ is chosen such that $f(1)=1 / 2$, or, equivalently

$$
2^{-\mathrm{n}} \Gamma(\mathrm{n}+1)^{-1} \int_{0}^{1}(1-t)^{-\mathrm{n}-1} \alpha_{f}(t) d t=1 .
$$

Consider a random variable $Z$ with the probability density function

$$
2^{-\mathrm{n}} \Gamma(\mathrm{n}+1)^{-1} s^{-\mathrm{n}-1} \alpha_{f}(1-s) \mathbb{1}_{[0,1]}(s),
$$

and $Z$ is independent of $r$. Then, for $\varphi:=\alpha_{f} / f$, 


$$
\int_{0}^{Z} \varphi(1-Z+v) d \mathrm{~L}_{v}(r) \stackrel{(\text { law })}{=} \mathcal{E}(1)
$$

Corollary 3.6. - If $Z \stackrel{\text { (law) }}{=} Z_{|\mathrm{n}|, \alpha}, \alpha>0$, is a beta random variable, independent of $r$, then,

$$
2^{\mathrm{n}+1} \Gamma(\mathrm{n}+1) \mathrm{B}(|\mathrm{n}|, \alpha)^{-1} \int_{0}^{Z}(1-Z+v)^{\mathrm{n}} d \mathrm{~L}_{v}(r) \stackrel{(\text { law })}{=} \mathcal{E}(1) .
$$

Proposition 3.7. - Consider a continuous function $\varphi:] 0,1] \rightarrow[0, \infty[$ such that $t^{|\mathrm{n}|} \varphi(t)$ converges as $t \downarrow 0$. For $\lambda>0$, we denote

$$
\Xi_{\varphi}(a):=\mathrm{E}_{0}^{\mathrm{n}}\left[\exp \left(-\lambda \int_{0}^{1-a} \varphi(a+v) d \mathrm{~L}_{v}(r)\right)\right], a \in[0,1] .
$$

Then,

$$
\left(I+\left(\lambda / c_{\mathrm{n}}\right) \mathcal{B}_{\varphi}\right) \Xi_{\varphi}=\mathbf{1}
$$

where, $c_{\mathrm{n}}$ is given by (2.12), and we denote, for a positive Borel function $h$,

$$
\left(\mathcal{B}_{\varphi} h\right)(a):=(1-a)^{\mathrm{n}+1} J^{-\mathrm{n}}\left(\tilde{\varphi} \epsilon_{-\mathrm{n}-1} \tilde{h}\right)(1-a), a \in[0,1]
$$

(recall that $\left.\epsilon_{\gamma}(s)=s^{\gamma}\right)$. Hence,

$$
\Xi_{\varphi}(a)=\sum_{k \geq 0}\left(-\lambda / c_{\mathrm{n}}\right)^{k}\left(\mathcal{B}_{\varphi}^{k} \mathbf{1}\right)(a), a \in[0,1]
$$

\section{PROOFS AND REMARKS}

Proof of Theorem 3.1. - (i) Let us denote $\psi(t):=t^{|\mathrm{n}|} \varphi(t)$. It is enough to consider (3.1) for $f$ and $\psi$ positive polynomials. Indeed, for general functions $f, \psi$ the result is obtained by a limiting procedure: there exist positive polynomials $f_{k}$ and $\psi_{k}$, such that

$$
f_{k} \rightarrow f, f_{k}^{\prime} \rightarrow f^{\prime}, \psi_{k} \rightarrow \psi
$$

uniformly, when $k \uparrow \infty$ (thus $\alpha_{f_{k}} \rightarrow \alpha_{f}$ ).

(ii) As said in the beginning of the previous section, to get (3.1) we need to 
verify the equalities (2.11) (combined with (2.6)) and (2.16). Suppose that, for given $f, \varphi$, the integral equation (2.16) has a solution, $\omega_{0}$. Then, section 2.1 asserts the existence of the solution $\omega(t, x)$ of the problem $(2.1)-(2.3)$, with boundary conditions $f$ and $\omega_{0}$. Moreover, by (2.8), we can find a function, which we denote, for the moment, as $\bar{\varphi}$. Since, by Proposition $2.4, \bar{\varphi}$ verifies (2.16) we conclude that $\bar{\varphi}=\varphi$. Finally, by Propositions 2.1-2.3 we get (2.11). Therefore, we need to study the integral equation (2.16).

(iii) By composition with $J^{- \text {n }}$ in (2.16), and using (1.14'), and (2.12'), we obtain

$$
\begin{gathered}
\Gamma(\mathrm{n}+1) f(t)+\left(\Gamma(|\mathrm{n}|) / 2^{\mathrm{n}+1}\right) J^{-\mathrm{n}}(\varphi f)(t) \\
=\Gamma(-\mathrm{n})^{-1} \int_{0}^{\infty} y \omega_{0}(y) d y \int_{0}^{t}(t-u)^{-\mathrm{n}-1} u^{\mathrm{n}-1} e^{-y^{2} / 2 u} d u
\end{gathered}
$$

(since $f(0)=0)$. Now we use the next equality

$$
\int_{0}^{t}(t-u)^{-\mathrm{n}-1} u^{\mathrm{n}-1} e^{-y^{2} / 2 u} d u=2^{-\mathrm{n}} \Gamma(-\mathrm{n}) t^{-\mathrm{n}-1} y^{2 \mathrm{n}} e^{-y^{2} / 2 t} .
$$

We can verify (b), by (1.8), (1.7) and (1.1'). Replacing (b) in (a) and using again (1.1') we obtain that (2.16) is equivalent to

$$
f(t)+\left(1 / c_{\mathrm{n}}\right) J^{-\mathrm{n}}(\varphi f)(t)=\Pi_{t}^{\mathrm{n}} \omega_{0}(0),
$$

We need to prove the existence of a solution $\omega_{0}$ for (c).

(iv) Let us note that, for given $f, \varphi$, such that $\varphi(t)=t^{\mathrm{n}} \psi(t)$, with $f, \psi$ positive polynomials, $J^{-\mathrm{n}}(\varphi f)$ is also a positive polynomial. By (1.13),

$$
\begin{gathered}
J^{-\mathrm{n}}(\varphi f)(t)=\Gamma(-\mathrm{n})^{-1} \int_{0}^{t}(t-u)^{-\mathrm{n}-1} u^{\mathrm{n}}(\psi f)(u) d u \\
=\Gamma(-\mathrm{n})^{-1} \int_{0}^{1}(1-v)^{-\mathrm{n}-1} v^{\mathrm{n}}(\psi f)(t v) d v
\end{gathered}
$$

and the right hand side is a positive polynomial in $t$.

(v) It suffices to prove the existence of a solution $\omega_{0}$ for

$$
\Pi_{t}^{\mathrm{n}} \omega_{0}(0)=\pi(t)
$$

where $\pi(t)$ is a positive polynomial, with $\pi(0)=0$. Since, for $p>0$,

$$
\Pi_{t}^{\mathrm{n}}\left(\epsilon_{2 p}\right)(0)=\mathrm{E}_{0}^{\mathrm{n}}\left(R_{t}^{2 p}\right)=t^{p} \mathrm{E}_{0}^{\mathrm{n}}\left(R_{1}^{2 p}\right),
$$


there is existence of a solution, $\omega_{0}(x)$ of the equation $\left(c^{\prime}\right)$, which is a polynomial in $x$, with $\omega_{0}(0)=0$.

Proof of Proposition 3.2. - First, we note that by the hypothesis on $f$, the function $\varphi$ satisfies the assumptions of Theorem 3.1. Take $\lambda>0$ and apply (3.1') with the function $\varphi=\alpha_{f} / f$ replaced by $\lambda \varphi$. Then, for every positive bounded Borel function $h$,

$$
\begin{aligned}
& \int_{0}^{1} \alpha_{f}(1-s) \mathrm{E}_{0}^{\mathrm{n}}\left[h\left(R_{s}\right) \exp \left(-\lambda \int_{0}^{s} \varphi(1-s+v) d \mathrm{~L}_{v}(R)\right)\right] d s \\
= & (1+\lambda)^{-1}\left(2^{\mathrm{n}+1} / \Gamma(|\mathrm{n}|)\right) \int_{0}^{\infty} y h(y) d y \int_{0}^{1} s^{\mathrm{n}-1} e^{-y^{2} / 2 s} f(1-s) d s .
\end{aligned}
$$

Since $1 /(1+\lambda)=\int_{0}^{\infty} \exp (-(1+\lambda) u) d u$, we obtain (3.2), noting that $h$ is arbitrary. Taking in the above equality $h \equiv 1$, by (2.13) and integrating with respect to $y$, we get on the right hand side $(1+\lambda)^{-1}$ (because $k_{f}=1$ ). We deduce (3.3) at once.

Proof of Proposition 3.3. - It is enough to prove that $\Lambda_{\varphi}$ verifies (3.5). We begin by proving that, for $l$ a regular function,

$$
\frac{\Gamma(|\mathrm{n}|)}{2^{\mathrm{n}+1}} \int_{0}^{1} \alpha_{\delta_{a}}(s) l(s) d s=(1-a)^{\mathrm{n}} l(1)+\Gamma(\mathrm{n}+1)\left(J^{\mathrm{n}+1} \tilde{l}^{\prime}\right)(1-a)
$$

(with, $\tilde{l}^{\prime}(s)=-l^{\prime}(1-s)$ ). Indeed, by $\left(2.12^{\prime}\right)$ and integrating by parts,

$$
\begin{gathered}
\left(\Gamma(|\mathrm{n}|) / 2^{\mathrm{n}+1}\right) \int_{0}^{1} \alpha_{f}(s) l(s) d s=\Gamma(\mathrm{n}+1) \int_{0}^{1} l(s)\left(J^{\mathrm{n}+1} f^{\prime}\right)(s) d s \\
=\int_{0}^{1} l(s) d s \int_{0}^{s}(s-u)^{\mathrm{n}} f^{\prime}(u) d u=\int_{0}^{1} f^{\prime}(u) d u \int_{u}^{1}(s-u)^{\mathrm{n}} l(s) d s \\
=\int_{0}^{1} f^{\prime}(u) d u\left[\frac{(1-u)^{\mathrm{n}+1}}{\mathrm{n}+1} l(1)-\int_{u}^{1} \frac{(s-u)^{\mathrm{n}+1}}{\mathrm{n}+1} l^{\prime}(s) d s\right] \\
=-\int_{0}^{1} f(u) d u\left[-(1-u)^{\mathrm{n}} l(1)+\int_{u}^{1}(s-u)^{\mathrm{n}} l^{\prime}(s) d s\right] .
\end{gathered}
$$

Taking $f:=\delta_{a}$, the Dirac delta function in the above equality, we get

$$
\begin{gathered}
\left(\Gamma(|\mathrm{n}|) / 2^{\mathrm{n}+1}\right) \int_{0}^{1} \alpha_{\delta_{a}}(s) l(s) d s=(1-a)^{\mathrm{n}} l(1)-\int_{a}^{1}(s-a)^{\mathrm{n}} l^{\prime}(s) d s \\
=(1-a)^{\mathrm{n}} l(1)-\int_{0}^{1-a}(1-a-v)^{\mathrm{n}} l^{\prime}(1-v) d v,
\end{gathered}
$$


from which we deduce (4.1).

Integrating with respect to $y$ in (3.1) (with $\lambda \varphi$ instead of $\varphi$ ), we obtain

$$
\left(\Gamma(|\mathrm{n}|) / 2^{\mathrm{n}+1}\right) \int_{0}^{1}\left(\alpha_{f}+\lambda \varphi f\right)(s) \Lambda_{\varphi}(s) d s=\int_{0}^{1}(1-s)^{\mathrm{n}} f(s) d s .
$$

Take now $l(s):=\Lambda_{\varphi}(s)$ and $f:=\delta_{a}$ and assume just a moment that $\Lambda_{\varphi}$ is regular. In this case, using (4.1), the above equality becomes:

$$
(1-a)^{\mathrm{n}} \Lambda_{\varphi}(1)+\Gamma(\mathrm{n}+1)\left(J^{\mathrm{n}+1} \tilde{\Lambda}_{\varphi}^{\prime}\right)(1-a)+\frac{\lambda \Gamma(|\mathrm{n}|)}{2^{\mathrm{n}+1}}\left(\varphi \Lambda_{\varphi}\right)(a)=(1-a)^{\mathrm{n}},
$$

or, since $\Lambda_{\varphi}(1)=1$,

$$
a^{\mathrm{n}}+\Gamma(\mathrm{n}+1)\left(J^{\mathrm{n}+1} \tilde{\Lambda}_{\varphi}^{\prime}\right)(a)+\left(\lambda \Gamma(|\mathrm{n}|) / 2^{\mathrm{n}+1}\right)\left(\tilde{\varphi} \tilde{\Lambda}_{\varphi}\right)(a)=a^{\mathrm{n}} .
$$

By composition with $J^{- \text {n }}$ and by (1.14), we obtain,

$$
\Lambda_{\varphi}(1-a)+\left(\lambda / c_{\mathrm{n}}\right) J^{-\mathrm{n}}\left(\tilde{\varphi} \tilde{\Lambda}_{\varphi}\right)(a)=1,
$$

(since $\left.J^{-\mathrm{n}}\left(\epsilon_{\mathrm{n}}\right)(a)=\Gamma(\mathrm{n}+1)\right)$. Moreover, this equality is true for continuous $\Lambda_{\varphi}$, so the regularity assumption can be removed.

REMARK 4.1. - Clearly, by (3.7) we get, for $a \in[0,1]$, and $k \in \mathbb{N}^{*}$,

$$
\mathrm{E}_{0}^{\mathrm{n}}\left[\left(\int_{0}^{1-a} \varphi(a+v) d \mathrm{~L}_{v}(R)\right)^{k}\right]=\left(k ! / c_{\mathrm{n}}^{k}\right)\left(\mathcal{A}_{\varphi}^{k} \mathbf{1}\right)(a) .
$$

If $a=0$, this expression for the moments is identical to (0.2), up to multiplication by 2 , since, by $(0.4), c_{\mathrm{n}} k_{\mathrm{n}} \Gamma(|\mathrm{n}|)=1$. The factor 2 is given by the different normalizations in (0.1) and (1.9); the local time defined in (0.1) is the double of that in (1.9).

REMARK 4.2. - We can prove the second part of Proposition 3.2, that is (3.3), as a consequence of Proposition 3.3. Indeed, we need to compute $\mathrm{E}\left[\Lambda_{\varphi}(1-Z)\right]$, where $Z$ is a random variable with the probability density function $\tilde{\beta}_{f}$ on $[0,1]$. We can write

$$
\begin{gathered}
\mathrm{E}\left[\left(\mathcal{A}_{\varphi} \Lambda_{\varphi}\right)(1-Z)\right]=\mathrm{E}\left[J^{-\mathrm{n}}\left(\tilde{\varphi} \tilde{\Lambda}_{\varphi}\right)(Z)\right] \\
=\Gamma(-\mathrm{n})^{-1} \int_{0}^{1} \alpha_{f}(1-s) d s \int_{0}^{s}(s-u)^{-\mathrm{n}-1}\left(\tilde{\varphi} \tilde{\Lambda}_{\varphi}\right)(u) d u \\
=\Gamma(-\mathrm{n})^{-1} \int_{0}^{1}\left(\tilde{\varphi} \tilde{\Lambda}_{\varphi}\right)(u) d u \int_{0}^{1-u}(1-u-v)^{-\mathrm{n}-1} \alpha_{f}(v) d v
\end{gathered}
$$




$$
\begin{gathered}
=\int_{0}^{1}\left(\varphi \Lambda_{\varphi}\right)(1-u)\left(J^{-\mathrm{n}} \alpha_{f}\right)(1-u) d u=c_{\mathrm{n}} \int_{0}^{1} \Lambda_{\varphi}(1-u)(\varphi f)(1-u) d u \\
=c_{\mathrm{n}} \int_{0}^{1} \Lambda_{\varphi}(1-u) \alpha_{f}(1-u) d u=c_{\mathrm{n}} \mathrm{E}\left[\Lambda_{\varphi}(1-Z)\right]
\end{gathered}
$$

(since $\left.\varphi f=\alpha_{f}\right)$. Taking the expectation in (3.5) we get $(1+\lambda) \mathrm{E}\left[\Lambda_{\varphi}(1-\right.$ $Z)]=1$. Therefore, $\mathrm{E}\left[\Lambda_{\varphi}(1-Z)\right]$ is the Laplace transform of $\mathcal{E}(1)$.

REMARK 4.3. - In the particular case $\varphi(t):=t^{\beta+\mathrm{n}}, \beta>0$, we can perform more calculations. Indeed, by (3.6) and induction, we get, for $k \in \mathbb{N}^{*}$,

$$
\left(\mathcal{A}_{\varphi}^{k} \mathbf{1}\right)(0)=\frac{1}{\Gamma(|\mathrm{n}|)^{k}} \frac{1}{k \beta} \prod_{j=1}^{k-1} \mathrm{~B}(j \beta,|\mathrm{n}|) .
$$

Therefore, we obtain the moments

$$
\mathrm{E}_{0}^{\mathrm{n}}\left[\left(\int_{0}^{1} v^{\beta+\mathrm{n}} d \mathrm{~L}_{v}(R)\right)^{k}\right]=k !\left(\frac{1}{2^{\mathrm{n}+1} \Gamma(\mathrm{n}+1)}\right)^{k} \frac{1}{k \beta} \prod_{j=1}^{k-1} \mathrm{~B}(j \beta,|\mathrm{n}|) .
$$

In [10] the case $\varphi(t):=t^{\mathrm{n}}$ was considered (see Sec. 2.3), and the results were obtained by a slightly different method. Explicit laws appeared for beta random variables $Z_{1, \alpha}, \alpha>0$.

REMARK 4.4. - We consider $\varphi(t):=(1-t)^{\beta}, \beta \geq \mathrm{n}$. Then we get, by (3.6) and induction, for $k \in \mathbb{N}^{*}$,

$$
\left(\mathcal{A}_{\varphi}^{k} \mathbf{1}\right)(a)=(1-a)^{k(\beta-\mathrm{n})} \prod_{j=1}^{k} \frac{\Gamma((\beta+1)+(j-1)(\beta-\mathrm{n}))}{\Gamma(j(\beta-\mathrm{n})+1)} .
$$

Therefore,

$$
\begin{gathered}
\mathrm{E}_{0}^{\mathrm{n}}\left[\left(\int_{0}^{1-a}(1-a-v)^{\beta} d \mathrm{~L}_{v}(R)\right)^{k}\right] \\
=\left(k ! / c_{\mathrm{n}}^{k}\right)(1-a)^{k(\beta-\mathrm{n})} \prod_{j=1}^{k} \frac{\Gamma((\beta+1)+(j-1)(\beta-\mathrm{n}))}{\Gamma(j(\beta-\mathrm{n})+1)} .
\end{gathered}
$$

REMARK 4.5. - If we take in (3.7') $\varphi=\mathbf{1}$ and $a=0$, by the above calculations with $\beta=0$, we obtain the moment expressions

$$
\mathrm{E}_{0}^{\mathrm{n}}\left[\left(c_{\mathrm{n}} \mathrm{L}_{1}(R)\right)^{k}\right]=k ! / \Gamma(k|\mathrm{n}|+1), k \in \mathbb{N}^{*}
$$


that is the moments of a Mittag-Leffler random variable with parameter $|\mathrm{n}|$ (see e.g. [7], p. 447 or [14], p. 129; see also [5], p. 452). This is equivalent with the well known fact that the subordinator $\left\{\tau_{t}: t \geq 0\right\}$ is stable with index $|\mathrm{n}|$ :

$$
\mathrm{E}_{0}^{\mathrm{n}}\left[e^{-\lambda \tau_{1}}\right]=e^{-c_{\mathrm{n}} \lambda^{|\mathrm{n}|}} .
$$

Here $\tau$ is the inverse of $\mathrm{L}(R), \tau_{t}:=\inf \left\{s: \mathrm{L}_{s}(R)>t\right\}$, and satisfies the scaling property:

$$
\left\{\tau_{t}: t \geq 0\right\} \stackrel{(\text { law })}{=}\left\{a \tau_{a^{\mathrm{n}} t}: t \geq 0\right\} .
$$

We can write, for any $k \in \mathbb{N}^{*}$,

$$
\mathrm{E}_{0}^{\mathrm{n}}\left[\left(c_{\mathrm{n}} \mathrm{L}_{1}(R)\right)^{k}\right]=\mathrm{E}_{0}^{\mathrm{n}}\left[c_{\mathrm{n}} / \tau_{1}^{k|\mathrm{n}|}\right]=c_{\mathrm{n}}^{k} \Gamma(k|\mathrm{n}|)^{-1} \int_{0}^{\infty} \lambda^{k|\mathrm{n}|-1} \mathrm{E}_{0}^{\mathrm{n}}\left[e^{-\lambda \tau_{1}}\right] d \lambda .
$$

By (4.4),

$$
\int_{0}^{\infty} \lambda^{k|\mathrm{n}|-1} \mathrm{E}_{0}^{\mathrm{n}}\left[e^{-\lambda \tau_{1}}\right] d \lambda=(k-1) ! /\left(|\mathrm{n}| c_{\mathrm{n}}^{k}\right)=\int_{0}^{\infty} \lambda^{k|\mathrm{n}|-1} e^{-c_{\mathrm{n}} \lambda^{|\mathrm{n}|}} d \lambda .
$$

and we deduce (4.5), using the injectivity of the Mellin transform. Assuming (4.5), the above equality gives (4.4).

Proof of Corollary 3.4. - We simply take in (4.3), $\beta=\mathrm{n}$ to obtain the $k$-moments and the Laplace transform of $\left(\Gamma(|\mathrm{n}|) / 2^{\mathrm{n}+1}\right) \mathcal{E}(1)$. Then, by scaling, we note that $\Lambda_{\varphi}$ does not depend on $a \in[0,1[$. The independence property announced in the corollary also follows by scaling.

Another proof of Corollary 3.4. - To prove (3.8), we may assume that $Z=1$, by scaling.

i) We denote $g:=g_{1}=\sup \left\{s \leq t: R_{s}=0\right\}$. It is sufficient to prove that

$$
A_{t}:=2^{\mathrm{n}+1} \Gamma(|\mathrm{n}|)^{-1} \int_{0}^{t}(1-v)^{\mathrm{n}} d \mathrm{~L}_{v}(R), t \in[0,1]
$$

is the dual predictable projection of $C_{t}:=\mathbb{1}_{\{g \leq t\}}, t \in[0,1]$, that is

$$
\mathrm{E}\left[h_{g}\right]=\mathrm{E}\left[\int_{0}^{1} h_{v} d A_{v}\right],
$$

for every predictable process $h \geq 0$ (see, e.g. [21], pp. 16-18). Indeed, 
we can follow the method in [2], p. 99. Since $A_{g}=A_{1}$, taking in (b) $h=\exp (-\lambda A)$, we have, for every $\lambda \geq 0$,

$$
\mathrm{E}\left[\exp \left(-\lambda A_{1}\right)\right]=\mathrm{E}\left[\int_{0}^{1} \exp \left(-\lambda A_{v}\right) d A_{v}\right]=\mathrm{E}\left[(1 / \lambda)\left(1-\exp \left(-\lambda A_{1}\right)\right)\right] .
$$

Thus, we obtain $\mathrm{E}\left[\exp \left(-\lambda A_{1}\right)\right]=1 /(1+\lambda)$, the desired result.

ii) We shall verify (b). It is enough to show this formula, for $h_{v}:=\mathbb{1}_{[0, T]}(v)$, where $T$ is a stopping time with values in $[0,1]$ :

$$
\mathrm{P}(g \leq T)=\mathrm{E}\left[A_{T}\right]
$$

Using (1.7), we can write

$$
\mathrm{P}_{x}\left(T_{0}>v\right)=2^{\mathrm{n}} \Gamma(|\mathrm{n}|)^{-1} \Phi\left(x^{2} / v\right),
$$

where we denoted $\Phi(y):=\int_{0}^{y} s^{-\mathrm{n}-1} e^{-s / 2} d s$. Then,

$$
\begin{gathered}
\mathrm{P}\left(g \leq T \mid \mathcal{F}_{T}\right)=2^{\mathrm{n}} \Gamma(|\mathrm{n}|)^{-1} \Phi\left(R_{T}^{2} /(1-T)\right) \\
=2^{\mathrm{n}} \Gamma(|\mathrm{n}|)^{-1} \Phi\left(X_{T} /(1-T)\right)=2^{\mathrm{n}} \Gamma(|\mathrm{n}|)^{-1} \Psi\left(R_{T}^{2|\mathrm{n}|} /(1-T)^{|\mathrm{n}|}\right)
\end{gathered}
$$

Here we denoted $\Psi(y):=\Phi\left(y^{1 /|\mathrm{n}|}\right)$ and by $\left\{X_{t}: 0 \leq t \leq 1\right\}$, the square of the Bessel process of index $\mathrm{n}$, whose infinitesimal generator is:

$$
\tilde{\mathcal{L}}=2 x\left(\partial^{2} / \partial x^{2}\right)+2(1+\mathrm{n})(\partial / \partial x) .
$$

Clearly, $(\tilde{\mathcal{L}}+(\partial / \partial s)) \Phi(x /(1-s))=0$. Using the fact that $X_{t}^{|\mathrm{n}|}-2|\mathrm{n}| \mathrm{L}_{t}(R)$ is a martingale (see section 1.3), by Ito's formula we can write

$\mathrm{E}\left[2^{\mathrm{n}} \Gamma(|\mathrm{n}|)^{-1} \Phi\left(X_{T} /(1-T)\right)\right]=\mathrm{E}\left[2^{\mathrm{n}} \Gamma(|\mathrm{n}|)^{-1} \int_{0}^{T} 2|\mathrm{n}| \Psi^{\prime}(0)(1-v)^{\mathrm{n}} d \mathrm{~L}_{v}(R)\right]$.

Since $\Psi^{\prime}(0)=1 /|\mathrm{n}|$, we get

$$
\mathrm{P}(g \leq T)=2^{\mathrm{n}+1} \Gamma(|\mathrm{n}|)^{-1} \mathrm{E}\left[\int_{0}^{T}(1-v)^{\mathrm{n}} d \mathrm{~L}_{v}(R)\right]=\mathrm{E}\left[A_{T}\right],
$$

by (a), and (c) is verified.

REMARK 4.6. - We can prove the following result which is, in some sense, a reciprocal result of Corollary 3.4: 
Assume that, for any $a \in[0,1[$, the integral

$$
\int_{0}^{1-a} \varphi(a+v) d \mathrm{~L}_{v}(R)
$$

is a standard exponential variable. Then

$$
\varphi(t)=\left(2^{\mathrm{n}+1} / \Gamma(|\mathrm{n}|)\right)(1-t)^{\mathrm{n}}
$$

For the proof, we note, by (3.4), that, for any $a \in\left[0,1\left[, \Lambda_{\varphi}(a)=1 /(1+\lambda)\right.\right.$. By Proposition $3.3, \Lambda_{\varphi}$ verifies (3.5). Hence $J^{-\mathrm{n}}(\tilde{\varphi})(a)=c_{\mathrm{n}}$. Since, by (1.15), $J^{-\mathrm{n}} \epsilon_{\mathrm{n}}=\Gamma(\mathrm{n}+1)$, we conclude using the injectivity of Abel's transform.

Proof of Proposition 3.5. - The right hand side of (3.1) can be written as

$$
\begin{gathered}
\left(2^{\mathrm{n}+1} / \Gamma(|\mathrm{n}|)\right) y \int_{0}^{1} s^{\mathrm{n}-1} e^{-y^{2} / 2 s} f(1-s) d s \\
=\left(2^{\mathrm{n}+1} / \Gamma(|\mathrm{n}|)\right) y^{2 \mathrm{n}+1} \int_{0}^{1 / y^{2}} u^{\mathrm{n}-1} e^{-1 / 2 u} f\left(1-u y^{2}\right) d u .
\end{gathered}
$$

We replace in (3.1) (with $\lambda \varphi$ instead of $\varphi$ ) the above equality, the expression $\left(1.1^{\prime}\right)$ for $p_{s}^{\mathrm{n}}(0, y)$ and we simplify by $y^{2 \mathrm{n}+1}$. Then, letting $y \downarrow 0$, we get

$$
\begin{gathered}
2^{-\mathrm{n}} \Gamma(\mathrm{n}+1)^{-1} \int_{0}^{1} \alpha_{f}(1-s) s^{-\mathrm{n}-1} \mathrm{E}_{0}^{\mathrm{n}}\left[\exp \left(-\lambda \int_{0}^{s} \varphi(1-s+v) d \mathrm{~L}_{v}(r)\right)\right] d s \\
=(1+\lambda)^{-1}\left(2^{\mathrm{n}+1} / \Gamma(|\mathrm{n}|)\right) f(1) \int_{0}^{\infty} u^{\mathrm{n}-1} e^{-1 / 2 u} d u
\end{gathered}
$$

(recall that $\left.\varphi f=\alpha_{f}\right)$. Since (recall that $f(1)=1 / 2$ )

$$
\left(2^{\mathrm{n}+1} / \Gamma(|\mathrm{n}|)\right) f(1) \int_{0}^{\infty} u^{\mathrm{n}-1} e^{-1 / 2 u} d u=2 f(1)=1,
$$

we get (3.10). To justify (3.9), we use (1.14') and (2.12'):

$$
\begin{gathered}
1=2\left(J^{-\mathrm{n}}\left(J^{\mathrm{n}+1} f^{\prime}\right)\right)(1)=2(\Gamma(|\mathrm{n}|))^{-1} \int_{0}^{1}(1-s)^{-\mathrm{n}-1}\left(J^{\mathrm{n}+1} f^{\prime}\right)(s) d s \\
=2^{-\mathrm{n}} \Gamma(\mathrm{n}+1)^{-1} \int_{0}^{1} s^{-\mathrm{n}-1} \alpha_{f}(1-s) d s .
\end{gathered}
$$


Proof of Corollary 3.6. - The result is obtained taking $f(t)=(1 / 2) t^{\alpha-\mathrm{n}-1}$ in Proposition 3.5.

Proof of Proposition 3.7. - Let us denote

$$
\Lambda_{\varphi}(a ; y):=\mathrm{E}_{0}^{\mathrm{n}}\left[\exp \left(-\lambda \int_{0}^{1-a} \varphi(a+v) d \mathrm{~L}_{v}(R)\right) \mid R_{1-a}=y\right]
$$

and

$$
l(s):=(1-s)^{-\mathrm{n}-1} e^{-y^{2} / 2(1-s)} \Lambda_{\varphi}(s ; y) .
$$

Clearly, $\Xi_{\varphi}(a)=\Lambda_{\varphi}(a ; 0)$ and, for $y=0, \tilde{l}=\epsilon_{-\mathrm{n}-1} \tilde{\Xi}_{\varphi}$. With these notations and using (1.1'), (3.1), with $\lambda \varphi$ instead of $\varphi$, can be written as

$$
\begin{aligned}
& 2^{-\mathrm{n}} \Gamma(\mathrm{n}+1)^{-1} \int_{0}^{1}\left(\alpha_{f}+\lambda \varphi f\right)(s) l(s) d s \\
= & \left(2^{\mathrm{n}+1} / \Gamma(|\mathrm{n}|)\right) y^{-2 \mathrm{n}} \int_{0}^{1} s^{\mathrm{n}-1} e^{-y^{2} / 2 s} f(1-s) d s .
\end{aligned}
$$

Taking in (c) $f:=\delta_{a}$, the Dirac delta function, and using (4.1), we obtain

$$
\begin{gathered}
2^{\mathrm{n}} \Gamma(\mathrm{n}+1)^{-1}(1-a)^{\mathrm{n}} l(1)+2^{\mathrm{n}}\left(J^{\mathrm{n}+1} \tilde{l}^{\prime}\right)(1-a)+(\lambda \Gamma(|\mathrm{n}|) / 2 \Gamma(\mathrm{n}+1))(\varphi l)(a) \\
=4^{\mathrm{n}} y^{-2 \mathrm{n}}(1-a)^{\mathrm{n}-1} e^{-y^{2} / 2(1-a)} .
\end{gathered}
$$

Since, by (b), $l(1)=0$, we get

$$
2^{\mathrm{n}}\left(J^{\mathrm{n}+1} \tilde{l}^{\prime}\right)(a)+(\lambda \Gamma(|\mathrm{n}|) /(2 \Gamma(\mathrm{n}+1)))(\tilde{\varphi} \tilde{l})(a)=4^{\mathrm{n}} y^{-2 \mathrm{n}} a^{\mathrm{n}-1} e^{-y^{2} / 2 a},
$$

or, by composition with $J^{-\mathrm{n}}$,

$$
\begin{aligned}
& \tilde{l}(a)+\left(\lambda / c_{\mathrm{n}}\right) J^{-\mathrm{n}}\left(\tilde{\varphi} \epsilon_{-\mathrm{n}-1} \tilde{\Lambda}_{\varphi}(\bullet ; y)\right)(a) \\
= & 2^{\mathrm{n}} \Gamma(-\mathrm{n})^{-1} y^{-2 \mathrm{n}} \int_{0}^{a}(a-u)^{-\mathrm{n}-1} u^{\mathrm{n}-1} e^{-y^{2} / 2 u} d u
\end{aligned}
$$

On the right hand side of $(\mathrm{d})$, we make the change of variable $u=v y^{2}$ to get

$$
\begin{gathered}
\tilde{l}(a)+\left(\lambda / c_{\mathrm{n}}\right) J^{-\mathrm{n}}\left(\tilde{\varphi} \epsilon_{-\mathrm{n}-1} \tilde{\Lambda}_{\varphi}(\bullet ; y)\right)(a) \\
=2^{\mathrm{n}} \Gamma(-\mathrm{n})^{-1} \int_{0}^{a / y^{2}}\left(a-v y^{2}\right)^{-\mathrm{n}-1} v^{\mathrm{n}-1} e^{-1 / 2 v} d v .
\end{gathered}
$$


Then, letting $y \downarrow 0$ in the above equality, we obtain,

$$
a^{-\mathrm{n}-1} \tilde{\Xi}_{\varphi}(a)+\left(\lambda / c_{\mathrm{n}}\right) J^{-\mathrm{n}}\left(\tilde{\varphi} \epsilon_{-\mathrm{n}-1} \tilde{\Xi}_{\varphi}\right)(a)=a^{-\mathrm{n}-1},
$$

and making a straightforward calculation on its right side hand, we get (3.12).

REMARK 4.7. - By (3.14) we get the following moment expressions: for $a \in[0,1]$, and $k \in \mathbb{N}^{*}$,

$$
\mathrm{E}_{0}^{\mathrm{n}}\left[\left(\int_{0}^{1-a} \varphi(a+v) d \mathrm{~L}_{v}(r)\right)^{k}\right]=\left(k ! / c_{\mathrm{n}}^{k}\right)\left(\mathcal{B}_{\varphi}^{k} \mathbf{1}\right)(a) .
$$

REMARK 4.8. - We take $\varphi(t):=t^{\beta+\mathrm{n}}, \beta>0$, Then we get, by (3.6) and induction, for $k \in \mathbb{N}^{*}$,

$$
\left(\mathcal{B}_{\varphi}^{k} \mathbf{1}\right)(0)=\frac{1}{\Gamma(|\mathrm{n}|)^{k}} \prod_{j=1}^{k} \mathrm{~B}(j \beta,|\mathrm{n}|) .
$$

Therefore, we obtain the moments

$$
\mathrm{E}_{0}^{\mathrm{n}}\left[\left(\int_{0}^{1} v^{\beta+\mathrm{n}} d \mathrm{~L}_{v}(r)\right)^{k}\right]=k !\left(\frac{1}{2^{\mathrm{n}+1} \Gamma(\mathrm{n}+1)}\right)^{k} \prod_{j=1}^{k} \mathrm{~B}(j \beta,|\mathrm{n}|) .
$$

REMARK 4.9. As in [10], Sec. 1.4, we can use the moment (or the Laplace transform) formulas to deduce some limit theorems. For example, by (4.6) we can prove that, for $\beta \downarrow 0$,

$$
\frac{2^{\mathrm{n}+1} \Gamma(\mathrm{n}+1)}{\sqrt{\delta_{\mathrm{n}}}}\left(\sqrt{\beta} \int_{0}^{1} v^{\beta+\mathrm{n}} d \mathrm{~L}_{v}(r)-\frac{1}{2^{\mathrm{n}+1} \Gamma(\mathrm{n}+1) \sqrt{\beta}}\right) \stackrel{(\text { law })}{\longrightarrow} \mathcal{N}(0,1) .
$$

Here, $\mathcal{N}(0,1)$ denotes the standard normal distribution and

$$
\delta_{\mathrm{n}}:=\left(\Gamma^{\prime}(1) / \Gamma(1)\right)-\left(\Gamma^{\prime}(|\mathrm{n}|) / \Gamma(|\mathrm{n}|)\right), x \mathrm{~B}(x,|\mathrm{n}|)=1+\delta_{\mathrm{n}} x+o(x), x \downarrow 0 .
$$

The proof of this result is similar to the proof of Theorem 1.27 in [10]. The same result can be obtained for the Bessel local time using (4.2): for $\beta \downarrow 0$,

$$
\frac{2^{\mathrm{n}+1} \Gamma(\mathrm{n}+1)}{\sqrt{\delta_{\mathrm{n}}}}\left(\sqrt{\beta} \int_{0}^{1} v^{\beta+\mathrm{n}} d \mathrm{~L}_{v}(R)-\frac{1}{2^{\mathrm{n}+1} \Gamma(\mathrm{n}+1) \sqrt{\beta}}\right) \stackrel{(\text { law })}{\longrightarrow} \mathcal{N}(0,1) .
$$


Remark 4.10. - Proposition 3.5 can be obtained as a consequence of Proposition 3.7. Consider $Z$ a random variable with density

$$
2^{-\mathrm{n}} \Gamma(\mathrm{n}+1)^{-1} s^{-\mathrm{n}-1} \alpha_{f}(1-s) \mathbb{1}_{[0,1]}(s),
$$

independent from the Bessel bridge $r$. We compute

$$
\begin{gathered}
\operatorname{E}\left[\left(\mathcal{B}_{\varphi} \Xi_{\varphi}\right)(1-Z)\right]=\mathrm{E}\left[Z^{\mathrm{n}+1} J^{-\mathrm{n}}\left(\tilde{\varphi} \epsilon_{-\mathrm{n}-1} \tilde{\Xi}_{\varphi}\right)(Z)\right] \\
=2^{-\mathrm{n}} \Gamma(\mathrm{n}+1)^{-1} \Gamma(-\mathrm{n}) \int_{0}^{1} \alpha_{f}(1-s) d s \int_{0}^{s}(s-u)^{-\mathrm{n}-1}\left(\tilde{\varphi} \tilde{\Xi}_{\varphi}\right)(u) u^{-\mathrm{n}-1} d u \\
=2^{-\mathrm{n}} \Gamma(\mathrm{n}+1)^{-1} \Gamma(-\mathrm{n}) \int_{0}^{1}\left(\tilde{\varphi} \tilde{\Xi}_{\varphi}\right)(u) u^{-\mathrm{n}-1} d u \int_{0}^{1-u}(1-u-v)^{-\mathrm{n}-1} \alpha_{f}(v) d v \\
=2^{-\mathrm{n}} \Gamma(\mathrm{n}+1)^{-1} \int_{0}^{1}\left(\varphi \Xi_{\varphi}\right)(1-u) u^{-\mathrm{n}-1}\left(J^{-\mathrm{n}} \alpha_{f}\right)(1-u) d u \\
=2 \Gamma(|\mathrm{n}|)^{-1} \int_{0}^{1} \Xi_{\varphi}(1-u) u^{-\mathrm{n}-1}(\varphi f)(1-u) d u \\
=2 \Gamma(|\mathrm{n}|)^{-1} \int_{0}^{1} \Xi_{\varphi}(1-u) u^{-\mathrm{n}-1} \alpha_{f}(1-u) d u=c_{\mathrm{n}} \mathrm{E}\left[\Xi_{\varphi}(1-Z)\right]
\end{gathered}
$$

(since $\left.\alpha_{f}=\varphi f\right)$. Then, we take the expectation in (3.12) and we deduce (3.10).

REMARK 4.11. - We can obtain (3.3) from (3.10) and vice-versa. Indeed, by $(1.10)$, it is not difficult to see that

$$
\left(\mathrm{L}_{t}(r)\right)_{t \in[0,1]} \stackrel{(\text { law })}{=}\left(g^{\mathrm{n}} \mathrm{L}_{g t}(R)\right)_{t \in[0,1]} .
$$

Here, we denote $g:=g_{1}=\sup \left\{s<1: R_{s}=0\right\}$ which is independent from $\left(g^{-1 / 2} R_{g t}: t \leq 1\right)$, hence from $\left(g^{\mathrm{n}} \mathrm{L}_{g t}(R): t \geq 0\right)$. From (4.8), we obtain that, for any positive Borel function $\psi$,

$$
g^{|\mathrm{n}|} \int_{0}^{1} \psi(g v) d \mathrm{~L}_{v}(r) \stackrel{(\mathrm{law})}{=} \int_{0}^{1} \psi(v) d \mathrm{~L}_{v}(R) .
$$

Recall that (see section 1.4) $g$ is a beta random variable with parameters $-\mathrm{n}, \mathrm{n}+1$. Then, by (3.14), to deduce (3.7) (both for $a=0$ ), we need to verify, for any $k \in \mathbb{N}^{*}$, 


$$
\left(\mathcal{A}_{\varphi}^{k} \mathbf{1}\right)(0)=\mathrm{B}(-\mathrm{n}, \mathrm{n}+1)^{-1} \int_{0}^{1} t^{-\mathrm{n}-1}(1-t)^{\mathrm{n}} t^{-k \mathrm{n}}\left(\mathcal{B}_{\varphi(t \bullet}^{k} \mathbf{1}\right)(0) d t .
$$

For $k=1$ we can write, by (3.13),

$$
\begin{gathered}
\mathrm{B}(-\mathrm{n}, \mathrm{n}+1)^{-1} \int_{0}^{1} t^{-\mathrm{n}-1}(1-t)^{\mathrm{n}} t^{-k \mathrm{n}}\left(\mathcal{B}_{\varphi(t)}^{k} \bullet \mathbf{1}\right)(0) d t=\mathrm{B}(-\mathrm{n}, \mathrm{n}+1)^{-1} \Gamma(-\mathrm{n})^{-1} \\
\times \int_{0}^{1} t^{-\mathrm{n}-1}(1-t)^{\mathrm{n}} t^{-\mathrm{n}} d t \int_{0}^{1}(1-u)^{-\mathrm{n}-1} \varphi(t(1-u)) u^{-\mathrm{n}-1} d u \\
=\mathrm{B}(-\mathrm{n}, \mathrm{n}+1)^{-1} \Gamma(-\mathrm{n})^{-1} \int_{0}^{1} v^{-\mathrm{n}-1} \varphi(v) d v \int_{v}^{1}(1-t)^{\mathrm{n}}(t-v)^{-\mathrm{n}-1} d t \\
=\Gamma(-\mathrm{n})^{-1} \int_{0}^{1} v^{-\mathrm{n}-1} \varphi(v) d v=\left(\mathcal{A}_{\varphi} \mathbf{1}\right)(0),
\end{gathered}
$$

as we can see by making the changes of variables $t(1-u)=v$ and $s=$ $(t-v) /(1-v)$. The same reasoning applies for arbitrary $k$. We leave to the reader the proof of the fact that (3.14) can be obtained by (3.7).

REMARK 4.12. - Assume that the conditioning is $\left\{R_{1-a}=y\right\}$, with arbitrary $y$. Then a functional equation, similar to (3.5), can be written, using (3.1):

$$
\left(I+\left(\lambda / c_{\mathrm{n}}\right) \mathcal{A}_{\varphi}\right) \Psi_{\varphi}(\bullet ; y)=\psi(\bullet ; y)
$$

where

$$
\begin{gathered}
\Psi_{\varphi}(a ; y):=p_{1-a}^{\mathrm{n}}(0, y) \\
\times \mathrm{E}_{0}^{\mathrm{n}}\left[\exp \left(-\lambda \int_{0}^{1-a} \varphi(a+v) d \mathrm{~L}_{v}(R)\right) \mid R_{1-a}=y\right]
\end{gathered}
$$

and

$$
\psi(a ; y):=\Gamma(\mathrm{n}+1)^{-1} \Gamma(-\mathrm{n})^{-1} y \int_{0}^{a}(a-u)^{-\mathrm{n}-1} u^{\mathrm{n}-1} e^{-y^{2} / 2 u} d u
$$

Therefore, we get a similar expression as $(3.7)$ for $\Psi_{\varphi}(a ; y)$ with $\psi(\bullet ; y)$ instead of the constant function $\mathbf{1}$.

REMARK 4.13. - Here, we give a probabilistic explanation for the appearance of Abel's integral operator, using the Bessel meander. In fact, we can state a reciprocal result of Theorem 3.1: 
Consider the continuous function $f:[0,1] \rightarrow\left[0, \infty\left[\right.\right.$, such that $\left.\left.f \in C^{1}(] 0,1\right]\right)$, $f(0)=0$, and assume that the random variable $Z$ is independent from the Bessel process $R$, having the probability density function $\beta(1-s) \mathbb{I}_{[0,1]}(s)$. Suppose that the random variable $R_{Z}$ has the probability density function given by

$$
k_{f}^{-1}\left(2^{\mathrm{n}+1} / \Gamma(|\mathrm{n}|)\right) y \mathbb{1}_{[0, \infty[}(y) \int_{0}^{1} s^{\mathrm{n}-1} e^{-y^{2} / 2 s} f(1-s) d s .
$$

Then, the function $\beta$ is an Abel transform of $f^{\prime}$. More precisely,

$$
\beta(t)=\alpha_{f}(t)=c_{\mathrm{n}}\left(J^{\mathrm{n}+1} f^{\prime}\right)(t)
$$

For the proof, we recall that, by (1.12) and (1.12'),

$$
R_{t} \stackrel{(\text { law })}{=} \sqrt{t-g_{t}} m_{t}(1)
$$

and $m_{t}(1)$ is independent of $g_{t}$, being Rayleigh distributed. Let us denote, for any positive bounded Borel function $h$,

$$
\Phi_{h}(s):=\int_{0}^{\infty} h(y \sqrt{s}) y e^{-y^{2} / 2} d y .
$$

Then, by (4.13),

$$
\int_{0}^{1} \tilde{\beta}(s) \mathrm{E}_{0}^{\mathrm{n}}\left[\Phi_{h}\left(s-g_{s}\right)\right] d s=k_{f}^{-1}\left(2^{\mathrm{n}+1} / \Gamma(|\mathrm{n}|)\right) \int_{0}^{1} s^{\mathrm{n}} \Phi_{h}(s) \tilde{f}(s) d s .
$$

Hence, the probability density function of the random variable $Z-g_{Z}$ is

$$
k_{f}^{-1}\left(2^{\mathrm{n}+1} / \Gamma(|\mathrm{n}|)\right) s^{\mathrm{n}} \tilde{f}(s) \mathbb{1}_{[0,1]}(s) .
$$

On the other hand $Z-g_{Z} \stackrel{\text { (law) }}{=} Z\left(1-g_{1}\right)$, with $g_{1}$ independent from $Z$. Recall that $1-g_{1}$ is a beta random variable with parameters $\mathrm{n}+1,-\mathrm{n}$. Hence,

(b) $\mathrm{E}\left[l\left(Z-g_{Z}\right)\right]=\mathrm{B}(\mathrm{n}+1,-\mathrm{n})^{-1} \int_{0}^{1} l(y) d y \int_{y}^{1} u^{\mathrm{n}}(1-u)^{-\mathrm{n}-1} \tilde{\beta}(y / u) d u$, for any positive bounded Borel function $l$ on $[0,1]$. Combining (a) and (b) we get, for $y \in[0,1]$, 
(c)

$$
\begin{gathered}
k_{f}^{-1}\left(2^{\mathrm{n}+1} / \Gamma(|\mathrm{n}|)\right)(1-y)^{\mathrm{n}} f(y) \\
=\Gamma(\mathrm{n}+1)^{-1} \Gamma(-\mathrm{n})^{-1} \int_{1-y}^{1} u^{\mathrm{n}}(1-u)^{-\mathrm{n}-1} \beta(1-(1-y) / u) d u .
\end{gathered}
$$

We make on the right hand side of (c) the change of variable $v=1-(1-y) / u$ and we get, after straightforward calculations,

$$
f(y)=\left(1 / c_{\mathrm{n}}\right)\left(J^{-\mathrm{n}} \beta\right)(y) .
$$

We get the same result by composition with $J^{-\mathrm{n}}$ in (4.14).

\section{APPENDIX \\ Formulae on integrals of Bessel local times}

We gather here the main results obtained in [10] (these are denoted between brackets) and in the present paper.

\section{Explicit laws}

We denote by $\mathrm{L}_{t}(B)$ and $\mathrm{L}_{t}(b)$ the local times at 0 of the Brownian motion $B$ starting from 0 , and of the Brownian bridge $b$; we denote by $\mathrm{L}_{t}(R)$ and $\mathrm{L}_{t}(r)$ the local times at 0 of the Bessel process $R$, of index $\left.\mathrm{n} \in\right]-1,0[$, starting from 0 , and of the Bessel bridge $r$. Here, $Z_{a, b}$ denotes a beta random variable with parameters $a, b$ independent of the process for which the local time is considered; in particular $U=Z_{1,1}$ is a uniform random variable on $[0,1]$ and $V=Z_{\frac{1}{2}, \frac{1}{2}}$ is an arcsine random variable. $\mathcal{E}(1)$ is the standard exponential distribution and $\gamma(2)$ is the gamma distribution of parameter 2 . $c$ denotes a normalisation constant. The last passage time in 0 before time 1 is denoted $g=\sup \left\{s \leq 1: B_{s}\right.$ or $\left.R_{s}=0\right\}$. $Z$ is independent of the process for which the local time is considered. $\lambda$ is a strictly positive parameter. If $f \in C^{1}\left(\mathbb{R}_{+}^{*}\right)$, with $f(0)=0$, we denote by $\alpha_{f}$ the function

$$
\alpha_{f}(t)=\left(2^{\mathrm{n}+1} / \Gamma(|\mathrm{n}|)\right) \int_{0}^{t}(t-u)^{\mathrm{n}} f^{\prime}(u) d u
$$

1) Brownian motion: 
(B1) $\frac{\sqrt{2 \pi}}{\mathrm{B}\left(\frac{1}{2}, \lambda\right)} \int_{0}^{Z_{1, \lambda}} \frac{d \mathrm{~L}_{t}(B)}{\sqrt{1-Z_{1, \lambda}+t}} \stackrel{(\text { law })}{=} \mathcal{E}(1)$

(B2) $\frac{\sqrt{2 \pi}}{\mathrm{B}\left(\lambda, \frac{1}{2}\right)} \int_{0}^{1} \frac{d \mathrm{~L}_{t}(B)}{\sqrt{t+\frac{Z_{\lambda, 1}}{1-Z_{\lambda, 1}}}} \stackrel{(\text { law })}{=} \sqrt{\frac{\pi}{2}} \int_{0}^{1} \frac{d \mathrm{~L}_{t}(B)}{\sqrt{t+\frac{U}{1-U}}} \stackrel{(\text { law })}{=} \mathcal{E}(1)$

(B3) $\frac{\sqrt{2 \pi}}{\mathrm{B}\left(\lambda, \frac{1}{2}\right)} \sqrt{1-Z_{\lambda, \frac{1}{2}}} \int_{0}^{\infty} \frac{d \mathrm{~L}_{t}(B)}{\sqrt{(1+t)\left(t+Z_{\lambda, \frac{1}{2}}\right)}} \stackrel{(\text { law })}{=} \mathcal{E}(1)$

(B4) $\quad \sqrt{\frac{2}{\pi}} \int_{0}^{1} \frac{d \mathrm{~L}_{t}(B)}{\sqrt{1-t}} \stackrel{(\text { law })}{=} \sqrt{\frac{2}{\pi}} \int_{1}^{\infty} \frac{d \mathrm{~L}_{t}(B)}{\sqrt{t(t-1)}} \stackrel{(\text { law })}{=} \mathcal{E}(1)[1.23$, A.16], see also [A]

(B5) $\quad a \int_{0}^{\infty} \frac{d \mathrm{~L}_{t}^{a}(B)}{t} \stackrel{(\mathrm{law})}{=} \mathcal{E}(1),\left(\mathrm{L}^{a}\right.$ local time at level $\left.\left.a\right) \quad[\mathrm{Rk} .1 .26, i v)\right]$

(B6) $\quad \sqrt{2} \mathrm{~L}_{1}(B) \stackrel{(\text { law })}{=} \sqrt{2}\left|B_{1}\right| \stackrel{(\text { law })}{=} \operatorname{Mittag-Leffler}\left(\frac{1}{2}\right)$

2) Brownian bridge:

(b1) $\frac{\sqrt{2 \pi}}{\mathrm{B}\left(\lambda, \frac{1}{2}\right)} \int_{0}^{1} \frac{d \mathrm{~L}_{t}(b)}{\sqrt{t+\frac{Z_{\lambda, \frac{1}{2}}}{1-Z_{\lambda, \frac{1}{2}}}}} \stackrel{(\text { law })}{=} \mathcal{E}(1)$

(b2) $\quad \sqrt{\frac{2}{\pi}} \int_{0}^{1} \frac{d \mathrm{~L}_{t}(b)}{\sqrt{t+\frac{V}{1-V}}} \stackrel{(\text { law })}{=} \sqrt{\frac{2}{\pi}} \int_{0}^{1} \frac{d \mathrm{~L}_{t}(b)}{\sqrt{\frac{1}{V}-t}} \stackrel{(\text { law })}{=} \mathcal{E}(1)$

$$
\sqrt{\frac{2}{\pi}} \int_{0}^{1} \frac{d \mathrm{~L}_{t}(b)}{\sqrt{t+\frac{1-g}{g}}} \stackrel{(\text { law })}{=} \mathcal{E}(1)\left(g \stackrel{(\text { law })}{=} Z_{\frac{1}{2}, \frac{1}{2}}\right)
$$

$$
\sqrt{\frac{2}{\pi}} \int_{0}^{1} \frac{d \mathrm{~L}_{t}(b)}{\sqrt{t+\frac{Z}{1-Z}}} \stackrel{(\mathrm{law})}{=} \gamma(2), Z \stackrel{(\mathrm{law})}{=} \frac{1}{c \pi} \frac{\log 1 / u}{\sqrt{u(1-u)}} \mathbb{I}_{[0,1]}(u)
$$


(b5) $\quad \frac{1}{\sqrt{\pi}} \int_{0}^{\frac{1}{2}} \frac{d \mathrm{~L}_{t}(b)}{\sqrt{\left(\frac{1}{2}-t\right)(1-t)}} \stackrel{(\text { law })}{=} \frac{1}{\sqrt{\pi}} \int_{\frac{1}{2}}^{1} \frac{d \mathrm{~L}_{t}(b)}{\sqrt{t\left(t-\frac{1}{2}\right)}} \stackrel{(\text { law })}{=} \mathcal{E}(1)$

(b6) $\quad \mathrm{L}_{1}(b) \stackrel{(\text { law })}{=} \sqrt{2 \mathcal{E}(1)} \quad[$ Rk.1.26,iv) $]$

3) Bessel process:

(R1) $2|\mathrm{n}| \int_{0}^{Z} \varphi(1-Z+t) d \mathrm{~L}_{t}(R) \stackrel{(\text { law })}{=} \mathcal{E}(1), Z \stackrel{(\text { law })}{=} c \alpha_{f}(1-u) \mathbb{I}_{[0,1]}(u)$

(R2) $\quad \frac{2^{\mathrm{n}+1} \Gamma(\mathrm{n}+1)}{\mathrm{B}(|\mathrm{n}|, \lambda)} \int_{0}^{Z_{1, \lambda}}\left(1-Z_{1, \lambda}+t\right)^{\mathrm{n}} d \mathrm{~L}_{t}(R) \stackrel{(\text { law })}{=} \mathcal{E}(1) \quad[2.37],(3.3)$

(R3) $\quad \frac{2^{\mathrm{n}+1} \Gamma(\mathrm{n}+1)}{\mathrm{B}(|\mathrm{n}|, \lambda)} \int_{0}^{\frac{Z_{1, \lambda}}{1-Z_{1, \lambda}}}(1+t)^{\mathrm{n}} d \mathrm{~L}_{t}(R) \stackrel{(\text { law })}{=} \mathcal{E}(1) \quad[2.37],(3.3)$

(R4) $\quad \frac{2^{\mathrm{n}+1}}{\Gamma(|\mathrm{n}|)} \int_{0}^{Z_{1, \mathrm{n}+1}}\left(1-Z_{1, \mathrm{n}+1}+t\right)^{\mathrm{n}} d \mathrm{~L}_{t}(R) \stackrel{(\text { law })}{=} \mathcal{E}(1) \quad[2.37],(3.3)$

(R5) $\frac{2^{\mathrm{n}+1}}{\Gamma(|\mathrm{n}|)} \int_{0}^{Z}(Z-t)^{\mathrm{n}} d \mathrm{~L}_{t}(R) \stackrel{(\text { law })}{=} \mathcal{E}(1)(\forall Z>0)$

(R6) $\frac{2^{\mathrm{n}+1}}{\Gamma(|\mathrm{n}|)} \int_{0}^{1}(1-t)^{\mathrm{n}} d \mathrm{~L}_{t}(R) \stackrel{(\text { law })}{=} \mathcal{E}(1)$

(R7) $\frac{2^{\mathrm{n}+1}}{\Gamma(|\mathrm{n}|)} \int_{0}^{g \text { or } 1}(1-g+t)^{\mathrm{n}} d \mathrm{~L}_{t}(R) \stackrel{(\text { law })}{=} \mathcal{E}(1) \quad\left(g \stackrel{(\text { law })}{=} Z_{|\mathrm{n}| \mathrm{n}+1}\right)$

(R8) $\quad \frac{2^{\mathrm{n}+1} \Gamma(\mathrm{n}+1)}{\Gamma(|\mathrm{n}|)} \mathrm{L}_{1}(R) \stackrel{(\text { law })}{=} \operatorname{Mittag-Leffler}(|\mathrm{n}|) \quad$ (4.3), see also [D - K]

4) Bessel bridge:

(r1) $\quad 2|\mathrm{n}| \int_{0}^{Z} \varphi(1-Z+t) d \mathrm{~L}_{t}(r) \stackrel{(\text { law })}{=} \mathcal{E}(1), Z \stackrel{\text { (law) }}{=} c \frac{u^{-\mathrm{n}-1} \alpha_{f}(1-u)}{2^{\mathrm{n}} \Gamma(\mathrm{n}+1)} \mathbb{1}_{[0,1]}(u)$ 
(r2) $\frac{2^{\mathrm{n}+1} \Gamma(\mathrm{n}+1)}{\mathrm{B}(|\mathrm{n}|, \lambda)} \int_{0}^{Z_{|\mathrm{n}|, \lambda}}\left(1-Z_{|\mathrm{n}|, \lambda}+t\right)^{\mathrm{n}} d \mathrm{~L}_{t}(r) \stackrel{(\mathrm{law})}{=} \mathcal{E}(1)$

$$
\frac{2^{\mathrm{n}+1} \Gamma(\mathrm{n}+1)}{\mathrm{B}(\lambda,|\mathrm{n}|)} \int_{0}^{1}\left(\frac{Z_{\lambda,|\mathrm{n}|}}{1-Z_{\lambda,|\mathrm{n}|}}+t\right)^{\mathrm{n}} d \mathrm{~L}_{t}(r) \stackrel{(\mathrm{law})}{=} \mathcal{E}(1)
$$

(r4) $\frac{2^{\mathrm{n}+1}}{\Gamma(|\mathrm{n}|)} \int_{0}^{1}\left(\frac{1-g}{g}+t\right)^{\mathrm{n}} d \mathrm{~L}_{t}(r) \stackrel{(\mathrm{law})}{=} \mathcal{E}(1) \quad\left(g \stackrel{(\mathrm{law})}{=} Z_{|\mathrm{n}|, \mathrm{n}+1}\right)$

5) Ornstein-Uhlenbeck process:

(O1) $\frac{\sqrt{2 \pi}}{\mathrm{B}\left(\lambda, \frac{1}{2}\right)} \int_{0}^{\infty} \frac{d \mathrm{~L}_{t}(O)}{\sqrt{\frac{e^{t}}{1-Z_{\lambda, \frac{1}{2}}}-1}} \stackrel{(\text { law })}{=} \mathcal{E}(1)$

(O2) $\quad \sqrt{\frac{2}{\pi}} \int_{0}^{\infty} \frac{\sqrt{V} d \mathrm{~L}_{t}(O)}{\sqrt{e^{t}-V}} \stackrel{(\mathrm{law})}{=} \mathcal{E}(1)$

\section{$k$-th moments}

Here, $k \in \mathbb{N}^{*} ; \beta>0 ; c_{\mathrm{n}}=2^{\mathrm{n}+1} \Gamma(\mathrm{n}+1) / \Gamma(|\mathrm{n}|)$. For $\varphi, h:[0,1] \rightarrow \mathbb{R}_{+}$Borel functions, we denote, for $a \in[0,1]$,

$$
\left(\mathcal{A}_{\varphi} h\right)(a)=\Gamma(|\mathrm{n}|)^{-1} \int_{0}^{1-a}(1-a-u)^{|\mathrm{n}|-1}(\varphi h)(1-u) d u
$$

and

$\left(\mathcal{B}_{\varphi} h\right)(a)=(1-a)^{\mathrm{n}+1} \Gamma(|\mathrm{n}|)^{-1} \int_{0}^{1-a}((1-a-u)(1-u))^{|\mathrm{n}|-1}(\varphi h)(1-u) d u$

1) Brownian motion:

$$
\mathrm{E}_{0}\left[\left(\sqrt{1-Z_{\lambda, 1}} \int_{0}^{1}\left(Z_{\lambda, 1}+\left(1-Z_{\lambda, 1}\right) t\right)^{\beta-1 / 2} d \mathrm{~L}_{t}(B)\right)^{k}\right]
$$




$$
=(k !)(2 \pi)^{-k / 2}(\lambda /(k \beta+\lambda)) \prod_{j=0}^{k-1} \mathrm{~B}(j \beta+\lambda, 1 / 2), \lambda>0
$$

(MB2) $\quad \mathrm{E}_{0}\left[\left(\int_{0}^{1} t^{\beta-1 / 2} d \mathrm{~L}_{t}(B)\right)^{k}\right]$

$$
=(k !)(2 \pi)^{-k / 2}(k \beta)^{-1} \prod_{j=1}^{k-1} \mathrm{~B}(j \beta, 1 / 2)
$$

(MB3) $\mathrm{E}_{0}\left[\left(\int_{0}^{1} t^{\beta-1 / 2} d \mathrm{~L}_{t}(B)\right)^{k}\right]$ $=2^{\beta k(k-1)} /\left((2 \pi)^{k / 2} \beta^{k} \prod_{j=1}^{k-1} C_{2 j \beta}^{j \beta}\right), \beta \in \mathbb{N}^{*} \quad[$ Rk.1.26,ii)]

(MB4) $\quad \mathrm{E}_{0}\left[\left(\int_{0}^{1} t^{\beta-1 / 2} d \mathrm{~L}_{t}(B)\right)^{k}\right]$

$$
=\frac{(k-1) !}{2^{k / 2} \beta} \frac{\Gamma(\beta)}{\Gamma\left((k-1) \beta+\frac{1}{2}\right)} \prod_{j=1}^{k-1} \prod_{i=1}^{\beta-\frac{1}{2}}(j \beta-i), \beta-\frac{1}{2} \in \mathbb{N} \quad[\text { Rk.1.26,ii) }]
$$

2) Brownian bridge:

$$
\begin{gathered}
\text { (Mb1) } \quad \mathrm{E}_{0}\left[\left(\sqrt{1-Z_{\lambda, 1 / 2}} \int_{0}^{1}\left(Z_{\lambda, 1 / 2}+\left(1-Z_{\lambda, 1 / 2}\right) t\right)^{\beta-1 / 2} d \mathrm{~L}_{t}(b)\right)^{k}\right] \\
=(k !)(2 \pi)^{-k / 2} \prod_{j=1}^{k} \mathrm{~B}(j \beta+\lambda, 1 / 2), \lambda>0
\end{gathered}
$$

(Mb2) $\quad \mathrm{E}_{0}\left[\left(\int_{0}^{1} t^{\beta-1 / 2} d \mathrm{~L}_{t}(b)\right)^{k}\right]$

$$
=(k !)(2 \pi)^{-k / 2} \prod_{j=1}^{k} \mathrm{~B}(j \beta, 1 / 2)
$$

(Mb3) $\quad \mathrm{E}_{0}\left[\left(\int_{0}^{1} t^{\beta-1 / 2} d \mathrm{~L}_{t}(b)\right)^{k}\right]$

$$
\left.\left.=2^{\beta k(k+1)} /\left((2 \pi)^{k / 2} \beta^{k}\right) \prod_{j=1}^{k} C_{2 j \beta}^{j \beta}\right), \beta \in \mathbb{N}^{*} \quad[\text { Rk.1.26, } i i)\right]
$$




$$
\begin{aligned}
& \text { (Mb4) } \quad \mathrm{E}_{0}\left[\left(\int_{0}^{1} t^{\beta-1 / 2} d \mathrm{~L}_{t}(b)\right)^{k}\right] \\
& =\left(k ! / 2^{k / 2}\right)\left(\Gamma(\beta) / \Gamma\left(k \beta+\frac{1}{2}\right)\right) \prod_{j=1}^{k} \prod_{i=1}^{\beta-\frac{1}{2}}(j \beta-i), \beta-\frac{1}{2} \in \mathbb{N} \quad[\text { Rk.1.26,ii) }]
\end{aligned}
$$

3) Bessel process:

$$
\begin{aligned}
(\mathrm{MR} 1) \quad \mathrm{E}_{0}^{\mathrm{n}}\left[\left(\int_{0}^{1-a} \varphi(a+t) d \mathrm{~L}_{t}(R)\right)^{k}\right] \\
=\left(k ! / c_{\mathrm{n}}^{k}\right)\left(\mathcal{A}_{\varphi}^{k} \mathbf{1}\right)(a), a \in[0,1]
\end{aligned}
$$

(MR2) $\quad \mathrm{E}_{0}^{\mathrm{n}}\left[\left(\int_{0}^{1-a}(1-a-t)^{\beta} d \mathrm{~L}_{t}(R)\right)^{k}\right]$

$$
=\left(k ! / c_{\mathrm{n}}^{k}\right)(1-a)^{k(\beta-\mathrm{n})} \prod_{j=1}^{k} \frac{\Gamma((\beta+1)+(j-1)(\beta-\mathrm{n}))}{\Gamma(j(\beta-\mathrm{n})+1)}, a \in[0,1[
$$

(MR3) $\quad \mathrm{E}_{0}^{\mathrm{n}}\left[\left(\int_{0}^{1} t^{\beta+\mathrm{n}} d \mathrm{~L}_{t}(R)\right)^{k}\right]$

$$
=k !\left(\frac{1}{2^{\mathrm{n}+1} \Gamma(\mathrm{n}+1)}\right)^{k} \frac{1}{k \beta} \prod_{j=1}^{k-1} \mathrm{~B}(j \beta,|\mathrm{n}|)
$$

(MR4) $\quad \mathrm{E}_{0}^{\mathrm{n}}\left[e^{-\lambda \tau_{t}}\right]=e^{-c_{\mathrm{n}} \lambda^{|\mathrm{n}|} t},\left(\tau_{t}\right.$ the inverse of $\left.\mathrm{L}_{t}(R)\right)$

4) Bessel bridge:

$$
\begin{gathered}
(\mathrm{Mr} 1) \quad \mathrm{E}_{0}^{\mathrm{n}}\left[\left(\int_{0}^{1-a} \varphi(a+t) d \mathrm{~L}_{t}(r)\right)^{k}\right] \\
=\left(k ! / c_{\mathrm{n}}^{k}\right)\left(\mathcal{B}_{\varphi}^{k} \mathbf{1}\right)(a), a \in[0,1] \\
(\mathrm{Mr} 2) \quad \mathrm{E}_{0}^{\mathrm{n}}\left[\left(\int_{0}^{1} t^{\beta+\mathrm{n}} d \mathrm{~L}_{t}(r)\right)^{k}\right] \\
=k !\left(\frac{1}{2^{\mathrm{n}+1} \Gamma(\mathrm{n}+1)}\right)^{k} \prod_{j=1}^{k} \mathrm{~B}(j \beta,|\mathrm{n}|)
\end{gathered}
$$




\section{Limit theorems}

Here $\delta_{\mathrm{n}}=\left(\Gamma^{\prime}(1) / \Gamma(1)\right)-\left(\Gamma^{\prime}(|\mathrm{n}|) / \Gamma(|\mathrm{n}|)\right), \delta_{-1 / 2}=\log 4$. We have the convergence in law to the standard normal distribution of:

(LB1) $\sqrt{\frac{\pi}{\log 2}}\left(\sqrt{\beta} \int_{0}^{1} v^{\beta-1 / 2} d \mathrm{~L}_{v}(B)-\frac{1}{\sqrt{2 \pi \beta}}\right)$, as $\beta \downarrow 0[\mathrm{Rk} .1 .28]$

(LB2) $\sqrt{\frac{\pi}{2 \log 2}}\left(\frac{1}{\sqrt{\log 1 / \varepsilon}} \int_{0}^{1} \frac{d \mathrm{~L}_{v}(B)}{\sqrt{\varepsilon+v}}-\sqrt{\frac{\log 1 / \varepsilon}{2 \pi}}\right)$, as $\varepsilon \downarrow 0[1.53]$

(LB3) $\sqrt{\frac{\pi}{2 \log 2}}\left(\frac{1}{\sqrt{\log 1 / a^{2}}} \int_{0}^{1} \frac{d \mathrm{~L}_{v}^{a}(B)}{\sqrt{v}}-\sqrt{\frac{\log 1 / a^{2}}{2 \pi}}\right)$, as $a \downarrow 0[1.56]$

(LB4) $\sqrt{\frac{\pi}{2 \log 2}}\left(\frac{1}{\sqrt{\log (t / s)}} \int_{s}^{t} \frac{d \mathrm{~L}_{v}(B)}{\sqrt{v}}-\sqrt{\frac{\log (t / s)}{2 \pi}}\right)$, as $s / t \rightarrow 0[1.58]$

(Lb1) $\sqrt{\frac{\pi}{\log 2}}\left(\sqrt{\beta} \int_{0}^{1} v^{\beta-1 / 2} d \mathrm{~L}_{v}(b)-\frac{1}{\sqrt{2 \pi \beta}}\right)$, as $\beta \downarrow 0[1.50]$

(Lb2) $\sqrt{\frac{\pi}{2 \log 2}}\left(\frac{1}{\sqrt{\log 1 / \varepsilon}} \int_{0}^{1} \frac{d \mathrm{~L}_{v}(b)}{\sqrt{\varepsilon+v}}-\sqrt{\frac{\log 1 / \varepsilon}{2 \pi}}\right)$, as $\varepsilon \downarrow 0$ [Rk.1.28]

$(\mathrm{LR} 1) \frac{2^{\mathrm{n}+1} \Gamma(\mathrm{n}+1)}{\sqrt{\delta_{\mathrm{n}}}}\left(\sqrt{\beta} \int_{0}^{1} v^{\beta+\mathrm{n}} d \mathrm{~L}_{v}(R)-\frac{1}{2^{\mathrm{n}+1} \Gamma(\mathrm{n}+1) \sqrt{\beta}}\right)$, as $\beta \downarrow 0\left(4.7^{\prime}\right)$

$(\operatorname{Lr} 1) \frac{2^{\mathrm{n}+1} \Gamma(\mathrm{n}+1)}{\sqrt{\delta_{\mathrm{n}}}}\left(\sqrt{\beta} \int_{0}^{1} v^{\beta+\mathrm{n}} d \mathrm{~L}_{v}(r)-\frac{1}{2^{\mathrm{n}+1} \Gamma(\mathrm{n}+1) \sqrt{\beta}}\right)$, as $\beta \downarrow 0$ 


\section{REFERENCES}

[1] J. AzÉmA, Quelques applications de la théorie générale des processus, Invent. Math. Vol. 18, 1972, pp. 293-336

[2] J. AzÉma and M. Yor, Une solution simple au problème de Skorokhod, in: C. Dellacherie, P.A. Meyer and M. Weil, eds. Sém. Prob. XIII (Lect. Notes Math., Vol. 721, Berlin Heidelberg New York: Springer 1979), pp. 90-115 and 625-633

[3] M. Baxter and D. Williams, Symmetry characterizations of certain distributions 1, Math. Proc. Camb. Phil. Soc., Vol. 111, 1992, pp. 387-399

[4] M. Baxter and D. Williams, Symmetry characterizations of certain distributions 2, Math. Proc. Camb. Phil. Soc., Vol. 112, 1992, pp. 599-611

[5] J. Bertoin and M. Yor, Some independence results related to the arcsine law, J. Theor. Prob., Vol. 9, 1996, pp. 447-458

[6] A.N. Borodin and P. Salminen, Handbook of Brownian motion: facts and formulae, Basel Boston Berlin: Birkhäuser 1996

[7] D.A. Darling and M. Kac, On occupation times for Markov processes, Trans. A.M.S., Vol. 84, 1957, pp. 444-458

[8] R.K. Getoor, The Brownian escape process, Ann. Prob., Vol. 7, 1979, pp. 864-867

[9] R. Gorenflo and S. Vessella, Abel integral equations: analysis and applications, Lect. Notes Math., Vol. 1461, Berlin Heidelberg New York: Springer 1991

[10] M. Gradinaru, B. Roynette, P. Vallois and M. Yor, The laws of Brownian local time integrals, Prépublication no. 38-1997, Institut Élie Cartan, Université Henri Poincaré (Vandœuvre-lès-Nancy, France); to appear in Computational and Applied Mathematics (1999)

[11] K. Ito and H.P. MCKean, Diffusion processes and their sample paths, Berlin Heidelberg New York: Springer 1965

[12] Th. Jeulin, Semi-martingales et grossissement d'une filtration, Lect. Notes Math., Vol. 833, Berlin Heidelberg New York: Springer 1980

[13] J. Kent, Some probabilistic properties of Bessel functions, Ann. Prob., Vol. 6, 1978, pp. $760-770$

[14] S.A. Molchanov and E. Ostrovskit, Symmetric stable processes as traces of degenerate diffusion processes, Th. Prob. Appl., Vol. 14, 1969, pp. 128-131

[15] J.W. Pitman and M. Yor, Bessel processes and infinitely divisible laws, in: D. Williams, ed. Stochastic Integrals (Lect. Notes Math., Vol. 851, Berlin Heidelberg New York: Springer 1981) pp. 285-370

[16] B. RAJEev and M. Yor, Local times and almost sure convergence of semi-martingales, Ann. Inst. "H. Poincaré", Vol. 31, 1995, pp. 653-667

[17] D. Revuz and M. Yor, Continuous martingales and Brownian motion, 3rd edition, Berlin Heidelberg New York: Springer 1999

[18] M.J. Sharpe, Some transformations of diffusions by time reversal, Ann. Prob., Vol. 8, 1980, pp. 1157-1162

[19] S. SATo and M. Yor, Computations of moments for discounted Brownian additive functionals, Prépublication no. 392-1997, Laboratoire. de Probabilités, Université Pierre et Marie Curie (Paris, France); to appear in J. Math. Kyoto (1998)

[20] M. Yor, Some aspects of Brownian motion I: Some special functionals, Basel Boston, Berlin: Birkhäuser 1992

[21] M. Yor, Local Times and Excursions for Brownian Motion: a concise introduction, Caracas: Universidad Central de Venezuela 1995

[22] M. Yor, Some aspects of Brownian motion II: Some recent martingale problems, 
Basel Boston, Berlin: Birkhäuser 1997

[23] M. Yor, On certain discounted arcsine laws, Stoch. Proc. and their Appl., Vol. 71, 1997, pp. 111-122 